\title{
Eunotia Ehrenberg (Bacillariophyceae) do rio Guaraguaçu, litoral do Paraná, Brasil
}

\author{
Priscila Izabel Tremarin ${ }^{1,2}$, Thelma Alvim Veiga Ludwig ${ }^{1}$ Hermes Moreira Filho ${ }^{1}$
}

Recebido em 25/04/2007. Aceito em 26/11/2007

\begin{abstract}
RESUMO - (Eunotia Ehrenberg (Bacillariophyceae) do rio Guaraguaçu, litoral do Paraná, Brasil). O trabalho apresenta o levantamento florístico da família Eunotiaceae do rio Guaraguaçu, um dos principais rios da bacia hidrográfica litorânea paranaense, região sul do Brasil. Amostras foram coletadas em abril e outubro de 2003 no plâncton e perifíton em seis estações ao longo do leito do rio. Identificaram-se 40 espécies, sendo 16 novos registros para o estado do Paraná: Eunotia anamargaritae, E. auriculata, E. batavica, E. convexa var. convexa f. impressa, E. dacostae, E. denticulata, E. fallax var. groenlandica, E. herzogii, E. meridiana, E. muscicola var. tridentula, E. nymanniana, E. pseudoindica, E. trinacria, Eunotia sp. 2, Eunotia sp. 3 e Eunotia sp. 4.
\end{abstract}

Palavras-chave: diatomáceas, taxonomia, bacia litorânea, plâncton, perifíton

ABSTRACT - (Eunotia Ehrenberg (Bacillariophyceae) of Guaraguaçu river, littoral of Paraná State, Brazil). The survey is a inventory floristic of Eunotiaceae family from Guaraguaçu river, one of the important lotic ecosystems of Litoranea watershed, State of Paraná, South Brazil. Samples were collected on April and October of 2003 from plankton and periphyton at six localities along the river. Forty species were identified and 16 taxa were registered for first time to State of Paraná: Eunotia anamargaritae, E. auriculata, E. batavica, E. convexa var. convexa f. impressa, E. dacostae, E. denticulata, E. fallax var. groenlandica, E. herzogii, E. meridiana, E. muscicola var. tridentula, E. nymanniana, E. pseudoindica, E. trinacria, Eunotia sp. 2, Eunotia sp. 3 and Eunotia sp. 4.

Key words: diatoms, taxonomy, littoral basin, plankton, periphyton

\section{Introdução}

Na América do Sul, trabalhos relevantes sobre a família Eunotiaceae foram desenvolvidos por Reichardt (1995) na Guiana Francesa e, De Oliveira \& SteinitzKannan (1992) e Sala et al. (2002) na Amazônia equatoriana e colombiana, respectivamente. Além destes, Metzeltin \& Lange-Bertalot (1998), em estudo sobre a flora diatomológica de regiões tropicais sul-americanas, registraram elevada riqueza de espécies do gênero Eunotia Ehrenberg, incluindo novidades taxonômicas para a ciência. Entre os estudos florísticos brasileiros, destacam-se os realizados por Patrick (1940a; b) no nordeste do Brasil e que descreveu cinco novas espécies para a família Eunotiaceae com base em amostras dos Estados do Pará, Maranhão, Ceará, Rio Grande do Norte, Paraíba, Pernambuco e Distrito Federal. Registros importantes foram realizados por Hustedt (1965), SouzaMozimann et al. (1997) e Ferrari et al. (2007) para a região amazônica, sendo que estes últimos determinaram 23 espécies de Eunotia, baseando-se em amostras provenientes de igarapés com $\mathrm{pH}$ ácido da Amazônia.
No Paraná trabalhos relevantes foram desenvolvidos por Ludwig \& Valente-Moreira (1989) que identificaram 24 espécies de Eunotia em amostras provenientes do lago do parque regional do Iguaçu, sendo cinco novos registros para o Estado, e Fürstenberger \& ValenteMoreira (2000), com duas novas citações, dentre as 11 espécies de Eunotia determinadas em amostras da lagoa Tarumã, localizada no município Ponta Grossa. Uma nova espécie de Eunotia, E. itapuana, foi descrita por Torgan \& Becker $(1997 ; 1998)$ ocorrendo em ambiente subaéreo na região costeira do Rio Grande do Sul. Ainda para o mesmo Estado, Torgan \& Delani (1988) registraram 35 táxons específicos e infra-específicos de Eunotia para um ecossistema pantanoso. Bicudo et al. (1999), em estudo da diatomoflórula da Reserva Biológica do Parque Estadual das Fontes do Ipiranga em São Paulo, identificaram 16 espécies pertencentes à ordem Eunotiales, sendo nove citações pioneiras para o Estado. O gênero Eunotia também foi bem representado no estudo sobre Thalassiosiraceae e Eunotiaceae em bancos de macrófitas aquáticas da lagoa Bonita, Distrito Federal (Souza \& Moreira Filho 1999), sendo que este

\footnotetext{
1 Universidade Federal do Paraná, Departamento de Botânica, Setor de Ciências Biológicas, Centro Politécnico, C. Postal 19031, 81531-990 Jardim das Américas, Curitiba, PR, Brasil

2 Autor para correspondência: ptremarin@gmail.com
} 
levantamento resultou na proposição de uma nova espécie do gênero (Souza \& Compère 1999).

Trabalho taxonômico envolvendo a flora de diatomáceas em rios no litoral do Paraná foi desenvolvido por Landucci \& Ludwig (2005), divulgando as Coscinodiscophyceae e Fragilariophyceae, mas estudos sobre Eunotiaceae são inexistentes na bacia hidrográfica Litorânea.

O presente estudo teve por objetivos dar continuidade ao inventário da diatomoflórula de ambientes lóticos do Estado do Paraná, contribuindo com a taxonomia e o registro geográfico das diatomáceas pertencentes à família Eunotiaceae do rio Guaraguaçu.

\section{Material e métodos}

O rio Guaraguaçu é um sistema litorâneo importante pela dimensão e volume d'água, apresentando influência do regime de marés uma vez que parte de seu leito situase em região de planície costeira. Está localizado na bacia hidrográfica Litorânea paranaense, abrangendo os municípios de Pontal do Paraná, Paranaguá e Matinhos. Coletas foram realizadas nos meses de abril e outubro/2003 em seis estações de coleta $\left(25^{\circ} 43^{\prime} \mathrm{S}\right.$ -

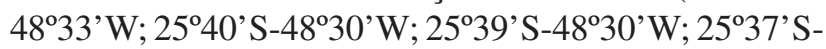
$48^{\circ} 30^{\prime} \mathrm{W} ; 25^{\circ} 36^{\prime} \mathrm{S}-48^{\circ} 29^{\prime} \mathrm{W}, 25^{\circ} 35^{\prime}$ 'S- $48^{\circ} 29^{\prime} \mathrm{W}$ ) durante período de maré alta e baixa, registrando-se os valores de salinidade durante a amostragem (Fig. 1). Vinte e nove amostras foram analisadas. As amostras de plâncton foram coletadas através de rede com abertura de malha de $25 \mu \mathrm{m}$ e as de perifíton pela raspagem de partes de macrófitas aquáticas submersas. Lâminas para estudo foram confeccionadas com material oxidado através da técnica de Simonsen (1974), modificada por MoreiraFilho \& Valente-Moreira (1981). As amostras foram registradas no Herbário da Universidade Federal do Paraná (UPCB). Ilustrações foram obtidas em fotomicroscópio Olympus BX40 com filme Imagelink (Kodak). O registro de ocorrência das espécies para o Estado foi baseado em trabalhos já publicados. As identificações basearam-se, principalmente, em Hustedt (1930), Krammer \& Lange-Bertalot (1991) e Metzeltin \& Lange-Bertalot (1998). O sistema de classificação baseou-se em Round et al. (1990) e a terminologia para descrição das espécies em Barber \& Haworth (1981). Descrições e comentários taxonômicos relevantes foram providenciados para as 16 espécies pioneiras para o Estado paranaense.

\section{Resultados e discussão}

O levantamento florístico das Eunotiaceae do rio Guaraguaçu permitiu a identificação de 44 táxons, sendo 41 espécies, cinco variedades e uma forma taxonômica.

O rio Guaraguaçu sofre influência do regime de marés. As estações de coleta mais próximas da foz do rio, durante a maré alta, apresentaram valores de salinidade 10 e 13, considerados salobros de acordo com o sistema de Veneza (Smayda 1983), mas durante a maré baixa os valores estimados foram zero.

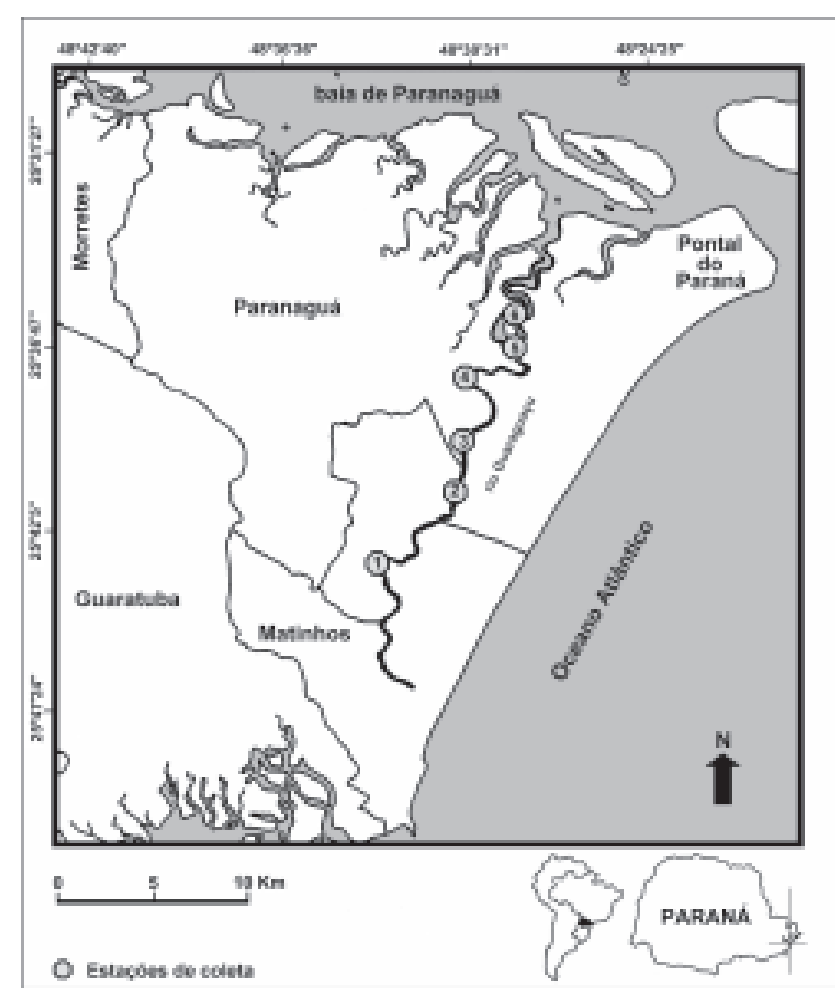

Figura 1. Localização das estações de amostragem no rio Guaraguaçu, litoral do Estado do Paraná, Brasil.

\section{Eunotia Ehrenberg}

\section{Chave de identificação para as espécies de Eunotia do rio Guaraguaçu}

1. Presença de ondulações na margem dorsal da valva

2. Valvas com mais de oito ondulações

33. E. serra

2. Valvas com menos de cinco ondulações

3. Extremidades destacadas do corpo valvar

4. Valvas com número par de ondulações dorsais 
5. Margem ventral reta a sutilmente côncava

6. Extremidades truncadas 26. E. praerupta var. bidens

6. Extremidades capitadas a subcapitadas

7. Comprimento superior a $39,2 \mu \mathrm{m}$

7. Comprimento inferior a $27,7 \mu \mathrm{m}$

8. Valvas com 16-17 estrias em $10 \mu \mathrm{m}$

8. Valvas com 12-14 estrias em $10 \mu \mathrm{m}$

14. E. herzogii

32. E. schneideri

5. Margem ventral fortemente côncava

9. Extremidades fletidas dorsalmente

41. Eunotia sp.2

9. Extremidades não fletidas dorsalmente

10. Ondulações achatadas

1. E. anamargaritae

10. Ondulações arredondadas...

39. E. zygodon

4. Valvas com número ímpar de ondulações dorsais

11. Valvas com mais de $5,5 \mu \mathrm{m}$ de largura

12. Extremidades largamente arredondadas

31. E. rabenhorstii

12. Extremidades de outras formas

13. Presença de estrias mais encurtadas na margem dorsal da valva, intercaladas com as demais

14. Ondulação mediana mais proeminente, longitudinalmente mais alongada que as demais

2. E. auriculata

14. Ondulação mediana mais proeminente ou não, longitudinalmente similar

as demais 36. E. trigibba

13. Ausência de estrias mais encurtadas na margem dorsal da valva

28. E. pyramidata var. pyramidata

11. Valvas com menos de $4 \mu \mathrm{m}$ de largura

15. Margem ventral côncava; extremidades capitadas

23. E. muscicola var. tridentula

15. Margem ventral reta a levemente côncava; extremidades subcapitadas 37. E. trinacria

3. Extremidades não destacadas do corpo valvar

29. E. pyramidata var. monodon

1. Ausência de ondulações na margem dorsal da valva

16. Extremidades mais largas que a largura mediana da valva

17. Margem dorsal e ventral reta

18. Extremidades largamente capitadas

12. E. flexuosa

18. Extremidades cuneadas 35. E. transfuga

17. Margem dorsal convexa e ventral côncava

19. Presença de intumescimento mediano na margem ventral da valva

13. E. formica

19. Ausência de intumescimento mediano na margem ventral da valva 3. E. batavica

16. Extremidades mais estreitas que a largura mediana da valva

20. Extremidades destacadas do corpo valvar

21. Valvas com extremidades fletidas dorsalmente

22. Extremidades subcapitadas

11. E. fallax var. groenlandica

22. Extremidades capitadas

23. Mais de 16 estrias em $10 \mu \mathrm{m}$

24. E. nymanniana

23. Menos de 12 estrias em $10 \mu \mathrm{m}$

24. Extremidades fortemente fletidas; nódulos terminais da rafe bem próximos às extremidades da valva 7. E. denticulata

24. Extremidades levemente fletidas; nódulos terminais da rafe não tão próximos das extremidades da valva 10. E. fallax var. fallax

21. Valvas sem extremidades fletidas dorsalmente

25. Aréolas conspícuas

26. Largura superior a $12,3 \mu \mathrm{m}$

25. E. praerupta var. praerupta

26. Largura inferior a $10,6 \mu \mathrm{m}$

27. Valvas com mais de 14 estrias em $10 \mu \mathrm{m}$

28. Extremidades cuneado-arredondadas

16. E. indica

28. Extremidades cuneadas

27. E. pseudoindica 
27. Valvas com menos de 12 estrias em $10 \mu \mathrm{m}$

29. Extremidades subcapitadas

22. E. monodon

29. Extremidades subcapitado-cuneadas

19. E. maior

25. Aréolas inconspícuas

30. Extremidades pouco destacadas do corpo valvar

31. Nódulos terminais da rafe grosseiros; extremidades largamente arredondadas

40. Eunotia sp. 1

31. Nódulos terminais da rafe mais delicados; extremidades subcapitadas a atenuado-arredondadas

34. E. sudetica

30. Extremidades bem destacadas do corpo valvar

32. Valvas com mais de $42,4 \mu \mathrm{m}$ compr.

33. Margem dorsal levemente convexa; 11-14 estrias em $10 \mu \mathrm{m}$

43. Eunotia sp. 4

33. Margem dorsal mais fortemente convexa; $15-18$ estrias em $10 \mu \mathrm{m}$

38. E. veneris

32. Valvas com menos de $21,3 \mu \mathrm{m}$ compr.

34. Extremidades rostrado-arredondadas, sutilmente fletidas para a margem ventral; 12-14 estrias em $10 \mu \mathrm{m}$

21. E. minor

34. Extremidades subcapitadas, não fletidas para a margem ventral; 20 estrias em $10 \mu \mathrm{m}$

8. E. exigua

20. Extremidades não destacadas do corpo valvar

35. Margem ventral intumescida próxima às extremidades da valva

36. Nódulos terminais projetados até aproximadamente metade da largura da extremidade valvar; extremidades atenuado-arredondadas 42. Eunotia sp. 3

36. Nódulos terminais restritos às margens da valva; extremidades arredondadas a atenuado-arredondadas 20. E. meridiana

35. Margem ventral não intumescida próxima às extremidades da valva

37. Extremidades arredondadas

38. Valvas assimétricas 9. E. faba

38. Valvas simétricas

39. Comprimento superior a $66,3 \mu \mathrm{m}$

40. Presença de uma área hialina próxima aos nódulos terminais que interrompe as estrias nas extremidades da valva 6. E. dacostae 40. Ausência desta característica 30. E. rabenhorstiana

39. Comprimento inferior a $20,5 \mu \mathrm{m}$

41. Largura valvar superior a $6,9 \mu \mathrm{m} ; 10-14$ em estrias $10 \mu \mathrm{m}$

18. E. luna var. globosa

41. Valvas com largura inferior a $4 \mu \mathrm{m} ; 12-16$ estrias em $10 \mu \mathrm{m}$ 17. E. intermedia

37. Extremidades atenuado-arredondadas

42. Nódulos terminais distantes das extremidades

15. E. incisa

42. Nódulos terminais próximos das extremidades 4. E. bilunaris

1. Eunotia anamargaritae Metzeltin \& Lange-Bertalot, Iconogr. Diatomol. 5: 51, pl. 37, fig. 9. 1998.

Eunotia zygodon var. elongata Hustedt in A. Schmidt,

Atl. Diatom. pl. 287, fig. 14. 1913.

Fig. 2

Valvas com margem dorsal convexa e duas ondulações pouco acentuadas, achatadas; margem ventral côncava; extremidades subcapitado-arredondadas, fletidas ventralmente, destacadas do corpo valvar, mais largas do que a largura mediana da valva; estrias paralelas a radiadas em direção às extremidades; aréolas

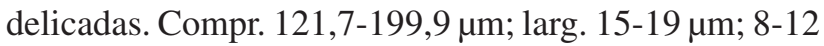

estrias em $10 \mu \mathrm{m} ; 20$-24 aréolas em $10 \mu \mathrm{m}$.

E. anamargaritae difere de E. zygodon não apenas em relação ao comprimento valvar, mas também no conjunto das demais características da valva, tal como a presença de ondulações achatadas na primeira espécie e arredondadas na segunda (Metzeltin \& Lange-Bertalot 1998).

Distribuição geográfica para o Estado do Paraná: primeira citação de ocorrência do táxon.

Ocorrência nas amostras: BRASIL. Paraná: Pontal do Paraná, rio Guaraguaçu, 16/IV/2003, fitoplâncton, P. Bigunas, T. Ludwig \& A. Campos s.n. (UPCB47493, 
47495, 47497, 47499); perifíton (UPCB47494, 47496, 47498, 47502, 47506); Matinhos, 24/X/2003, fitoplâncton, P. Bigunas, T. Ludwig \& J. Silva s.n. (UPCB47509); perifíton (UPCB47510, 47511); Pontal do Paraná, 24/X/2003, fitoplâncton, P. Bigunas, T. Ludwig \& J. Silva s.n. (UPCB47524, 47525).

2. Eunotia auriculata Grunow in Van Heurck, Syn. Diat.

Belg. pl. 35, fig. 15. 1881.

Fig. 3

Valvas com margem dorsal convexa apresentando três ondulações, ondulação mediana mais larga e proeminente que as demais; margem ventral côncava; extremidades truncadas, destacadas do corpo valvar, mais estreitas que a largura mediana da valva; estrias paralelas a radiadas em direção às extremidades, estrias encurtadas intercaladas com as demais, presentes na margem dorsal; aréolas inconspícuas. Compr. 45,8 $\mu$ m; larg. 13,4 $\mu \mathrm{m} ; 10$ estrias em $10 \mu \mathrm{m}$.

Distribuição geográfica para o Estado do Paraná: primeira citação de ocorrência do táxon.

Ocorrência nas amostras: BRASIL. Paraná: Matinhos, rio Guaraguaçu, 24/X/2003, perifíton, P. Bigunas, T. Ludwig \& J. Silva s.n. (UPCB47510).

3. Eunotia batavica Berg, Bot. Nat. p. 462, pl. 5, fig. 186. 1939.

Fig. 4-5

Valvas com margem dorsal levemente convexa; margem ventral com concavidade um pouco mais pronunciada; extremidades cuneado-arredondadas, não destacadas do corpo valvar; linha hialina longitudinal estreita no lado ventral da valva; estrias paralelas a radiadas nas extremidades; aréolas delicadas. Compr. 49,8-100,3 $\mu \mathrm{m}$; larg. 7,9-10,3 $\mu \mathrm{m} ; 12-16$ estrias em $10 \mu \mathrm{m} ; 12$ aréolas em $10 \mu \mathrm{m}$.

Distribuição geográfica para o Estado do Paraná: primeira citação de ocorrência do táxon.

Ocorrência nas amostras: BRASIL. Paraná: Pontal do Paraná, rio Guaraguaçu, 16/IV/2003, perifíton, P. Bigunas, T. Ludwig \& A. Campos s.n. (UPCB47494); Pontal do Paraná, 24/X/2003, perifíton, P. Bigunas, T. Ludwig \& J. Silva s.n. (UPCB47511).

4. Eunotia bilunaris (Ehrenberg) Mills, Index Gen. Spec. Diat. Syn. (2): 675. 1934.

Synedra bilunaris Ehrenberg, Abh. Akad. Wiss. Berl. p. 87.1831 (1832).

Fig. 6-9

Compr. 9,5-86,9 $\mu \mathrm{m}$; larg. 2,4-4 $\mu \mathrm{m} ; 16-22$ estrias em $10 \mu \mathrm{m}$.

Distribuição geográfica para o Estado do Paraná:
Cascavel (Tavares \& Valente-Moreira 2000), Curitiba (Ludwig \& Valente-Moreira 1989; Ludwig et al. 2005), Mangueirinha e Palmas (Ludwig \& Flôres 1995), Ponta Grossa (Fürstenberger \& Valente-Moreira 2000).

Ocorrência nas amostras: BRASIL. Paraná: Pontal do Paraná, rio Guaraguaçu, 16/IV/2003, fitoplâncton, P. Bigunas, T. Ludwig \& A. Campos s.n. (UPCB47493, 47495, 47497, 47499); perifíton (UPCB47494, 47496, 47500, 47502, 47503, 47506); Matinhos, 24/X/2003, fitoplâncton, P. Bigunas, T. Ludwig \& J. Silva s.n. (UPCB47509); perifíton (UPCB47510, 47511); Pontal do Paraná, 24/X/2003, fitoplâncton, P. Bigunas, T. Ludwig \& J. Silva s.n. (UPCB47512, 47514, 47523, 47524, 47516, 47519, 47525, 47521); perifíton (UPCB47513, 47515, 47517, 47518, 47520).

5. Eunotia convexa var. convexa f. impressa Hustedt, Ber. Deustch. Bot. Ges. (65): 133-144, pl. 141, fig. 7. 1952. Fig. 10

Valvas com margem dorsal convexa apresentando quatro ondulações, depressão mediana um pouco mais pronunciada que as demais; margem ventral reta; extremidades capitadas, destacadas do corpo valvar, mais estreitas que a largura mediana da valva; estrias paralelas a radiadas em direção às extremidades; aréolas inconspícuas. Compr. 39,2-47,4 $\mu \mathrm{m}$; larg. 4-5,5 $\mu \mathrm{m}$; 14-16 estrias em $10 \mu \mathrm{m}$.

Distribuição geográfica para o Estado do Paraná: primeira citação de ocorrência do táxon.

Ocorrência nas amostras: BRASIL. Paraná: Pontal do Paraná, rio Guaraguaçu, 16/IV/2003, fitoplâncton, $P$. Bigunas, T. Ludwig \& A. Campos s.n. (UPCB47493, 47495, 47497, 47499); perifíton (UPCB47494, 47496); 24/X/2003, fitoplâncton, P. Bigunas, T. Ludwig \& J. Silva s.n. (UPCB47516, 47523, 47525); perifíton (UPCB47513).

6. Eunotia dacostae Lange-Bertalot \& Metzeltin, Iconogr. Diatomol. 5: 56, pl. 50, fig. 5-6. 1998.

Fig. 11-12

Valvas com margem dorsal levemente convexa; margem ventral côncava; extremidades arredondadas, não destacadas do corpo valvar; estrias paralelas a radiadas nas extremidades, interrompidas nas extremidades por uma área hialina projetada dos nódulos terminais; aréolas inconspícuas. Compr. 66,3-143,5 $\mu$ m; larg. 6,3-7,8 $\mu \mathrm{m} ; 14-20$ estrias em $10 \mu \mathrm{m}$.

E. dacostae apresenta certa similaridade com E. parallela Ehrenberg, contudo a primeira apresenta menor curvatura valvar e uma área hialina próxima aos nódulos terminais que parte em direção à superfície valvar interrompendo as estrias nesta região.

Apesar da variação métrica da população estudada no rio Guaraguaçu apresentar-se um pouco inferior à 
registrada na obra original (compr. 100-120 $\mu \mathrm{m}$, larg. 7,5-8 $\mu \mathrm{m}$ e 13-14 estrias em $10 \mu \mathrm{m}$ ), os exemplares apresentaram as características diagnósticas da espécie.

Distribuição geográfica para o Estado do Paraná: primeira citação de ocorrência do táxon.

Ocorrência nas amostras: BRASIL. Paraná: Pontal do Paraná, rio Guaraguaçu, 16/IV/2003, perifíton, P. Bigunas, T. Ludwig \& A. Campos s.n. (UPCB47496); Matinhos, 24/X/2003, perifíton, P. Bigunas, T. Ludwig \& J. Silva s.n. (UPCB47510); Pontal do Paraná, 24/X/2003, perifíton, P. Bigunas, T. Ludwig \& J. Silva s.n. (UPCB47513).

7. Eunotia denticulata (Brébisson) Rabenhorst, Fl. Eur. Alg. 1(1): 73. 1864.

Himantidium denticulatum Brébisson in Kützing, Sp. Alg.

p. $10,1849$.

Fig. 13

Valvas com margem dorsal levemente convexa; margem ventral côncava; extremidades capitadas, fletidas dorsalmente, destacadas do corpo valvar, mais estreitas que a largura mediana da valva; estrias paralelas a sutilmente radiadas nas extremidades; aréolas inconspícuas.

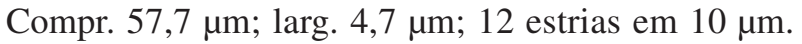

Distribuição geográfica para o Estado do Paraná: primeira citação de ocorrência do táxon.

Ocorrência nas amostras: BRASIL. Paraná: Pontal do Paraná, rio Guaraguaçu, 16/IV/2003, perifíton, P. Bigunas, T. Ludwig \& A. Campos s.n. (UPCB47502).

8. Eunotia exigua (Brébisson ex Kützing) Rabenhorst, Eur. Alg. sect. 1, p. 73. 1864.

Himantidium exiguum Brébisson ex Kützing, Sp. Alg., p. 8. 1849.

Fig. 14-15

Compr. 10,3-16,6 $\mu \mathrm{m}$; larg. 3,2-4 $\mu \mathrm{m} ; 20$ estrias em $10 \mu \mathrm{m}$.

Distribuição geográfica para o Estado do Paraná: Curitiba (Moreira Filho et al. 1973; Contin 1990; Ludwig et al. 2005), Mangueirinha, Palmas e Pinhão (Ludwig \& Flôres 1995), São José dos Pinhais (Momoli 1967).

Ocorrência nas amostras: BRASIL. Paraná: Pontal do Paraná, rio Guaraguaçu, 16/IV/2003, perifíton, P. Bigunas, T. Ludwig \& A. Campos s.n. (UPCB47494, 47498); 24/X/2003, fitoplâncton, P. Bigunas, T. Ludwig \& J. Silva s.n. (UPCB47512, 47516, 47523, 47526); perifíton (UPCB47513, 47515).

9. Eunotia faba (Ehrenberg) Grunow in Van Heurck, Syn. Diat. Belg. pl. 34, fig. 34. 1881.

Himantidium faba Ehrenberg, Abh. Akad. Wiss. Berl. pl.1/2, fig. 3. 1854.

Fig. 16
Compr. 25,6 $\mu \mathrm{m}$; larg. 7,5 $\mu \mathrm{m} ; 12$ estrias em $10 \mu \mathrm{m}$.

Distribuição geográfica para o Estado do Paraná: Curitiba (Ludwig et al. 2005), Pinhão (Ludwig \& Flôres 1995), Ponta Grossa (Fürstenberger \& Valente-Moreira 2000).

Ocorrência nas amostras: BRASIL. Paraná: Pontal do Paraná, rio Guaraguaçu, 24/X/2003, fitoplâncton, P. Bigunas, T. Ludwig \& J. Silva s.n. (UPCB47512).

10. Eunotia fallax A. Cleve var.fallax, Sven. Vet. Akad. Handl. 21(3): 33, pl. 1, fig. 35. 1895.

Fig. $17-18$

Compr. 31,9-52,5 $\mu \mathrm{m}$; larg.: 3,8-4,4 $\mu \mathrm{m}$; 11-12 estrias em $10 \mu \mathrm{m}$.

Distribuição geográfica para o Estado do Paraná: Curitiba (Contin 1990).

Ocorrência nas amostras: BRASIL. Paraná: Pontal do Paraná, rio Guaraguaçu, 24/X/2003, fitoplâncton, P. Bigunas, T. Ludwig \& J. Silva s.n. (UPCB47512, 47514).

11. Eunotia fallax var. groenlandica (Grunow) LangeBertalot \& Nörpel in Pascher, Süssw. Fl. Mitteur. 2: 207, pl. 150, fig. 10-15. 1991.

Eunotia paludosa Grunow var. groenlandica Grunow in Van Heurck, Syn. Diat. Belg. 1881.

Fig. 19-21

Valvas com margem dorsal convexa; margem ventral reta a sutilmente côncava; extremidades subcapitadas, sutilmente fletidas dorsalmente, pouco destacadas do corpo valvar, mais estreitas que a largura mediana da valva; estrias paralelas; aréolas inconspícuas. Compr.

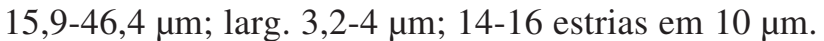

Krammer \& Lange-Bertalot (1991) sinonimizaram a var. gracillima Krasske com a var. groenlandica. Jensen (1985) comenta que E. fallax var. gracillima difere da variedade típica da espécie por apresentar extremidades não capitadas, valvas mais estreitas e extremidades geralmente pouco fletidas dorsalmente. Alguns exemplares de E. glacialis Meister, contidos em Krammer \& Lange-Bertalot (1991), lembram E. fallax var. groenlandica. Todavia, E. glacialis distingue-se por ter valvas sempre arqueadas, com margens recurvadas próximas às extremidades, além de poder apresentar extremidades capitadas (Patrick \& Reimer 1966).

Distribuição geográfica para o Estado do Paraná: primeira citação de ocorrência do táxon.

Ocorrência nas amostras: BRASIL. Paraná: Pontal do Paraná, rio Guaraguaçu, 16/IV/2003, fitoplâncton, P. Bigunas, T. Ludwig \& A. Campos s.n. (UPCB47501); perifíton (UPCB47502); Matinhos, 24/X/2003, perifíton, P. Bigunas, T. Ludwig \& J. Silva s.n. (UPCB47510, 47511); Pontal do Paraná, 24/X/2003, fitoplâncton, 

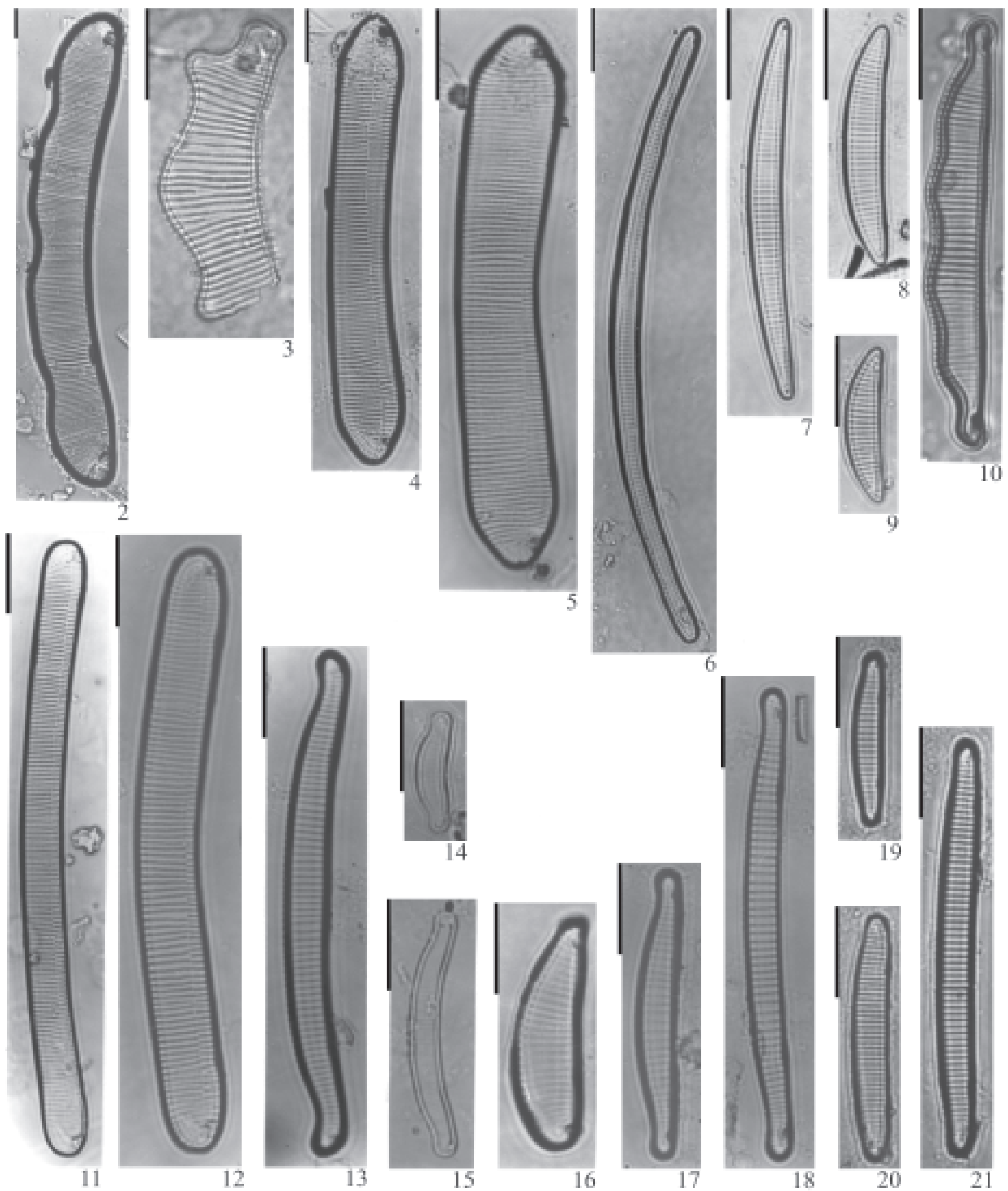

Figuras 2-21. Eunotia Ehrenberg (Bacillariophyceae) do rio Guaraguaçu, Paraná, Brasil. 2. Eunotia anamargaritae Metzeltin \& LangeBertalot. 3. Eunotia auriculata Grunow. 4-5. Eunotia batavica Berg. 6-9. Eunotia bilunaris (Ehrenberg) Mills. 10. Eunotia convexa var. convexa f. impressa Hustedt. 11-12. Eunotia dacostae Lange-Bertalot \& Metzeltin. 13. Eunotia denticulata (Brébisson) Rabenhorst. 14-15. Eunotia exigua (Brébisson ex Kützing) Rabenhorst. 16. Eunotia faba (Ehrenberg) Grunow. 17-18. Eunotia fallax A. Cleve var. fallax. 19-21. Eunotia fallax var. groenlandica (Grunow) Lange-Bertalot \& Nörpel. Barras $=10 \mu \mathrm{m}$. 
P. Bigunas, T. Ludwig \& J. Silva s.n. (UPC47512, 47514, 47516, 47523, 47524, 47525, 47521); perifíton (UPCB47513, 47517, 47520).

12. Eunotia flexuosa Brébisson ex Kützing, Sp. Alg. p. 6. 1949.

Fig. 22

Compr. 124-186 $\mu \mathrm{m}$; larg. 5,5-6 $\mu \mathrm{m}$; $14-18$ estrias em $10 \mu \mathrm{m}$.

Distribuição geográfica para o Estado do Paraná: Caiobá (Valente-Moreira \& Moreira Filho 1982; Moreira Filho \& Valente-Moreira 1984), Cascavel (Tavares \& Valente-Moreira 2000), Curitiba (Moreira Filho \& Momoli 1966; Moreira Filho et al. 1973; Ludwig \& ValenteMoreira 1989; Contin 1990; Lozovei \& Shirata 1990), Londrina (Bittencourt-Oliveira 2002), Palmeira (ValenteMoreira 1975), Ponta Grossa (Moro et al. 1994; Fürstenberger \& Valente-Moreira 2000; BittencourtOliveira 2002), São José dos Pinhais (Momoli 1967), Sapopema, Sertanópolis e Telêmaco Borba (BittencourtOliveira 2002).

Ocorrência nas amostras: BRASIL. Paraná: Pontal do Paraná, rio Guaraguaçu, 16/IV/2003, fitoplâncton, P. Bigunas, T. Ludwig \& A. Campos s.n. (UPCB47495, 47497, 47499); perifíton (UPCB47496, 47500); 24/X/2003, fitoplâncton, P. Bigunas, T. Ludwig \& J. Silva s.n. (UPCB47512).

13. Eunotia formica Ehrenberg, Phys. Abh. Akad. Wiss. Berl. 1: 414. 1841 (1843).

Fig. 23

Compr. 169,2-188 $\mu \mathrm{m}$; larg. 9,4-11,9 $\mu \mathrm{m} ; 8-10$ estrias em $10 \mu \mathrm{m} ; 22-26$ aréolas em $10 \mu \mathrm{m}$.

Distribuição geográfica para o Estado do Paraná: Curitiba (Contin 1990; Lozovei \& Shirata 1990).

Ocorrência nas amostras: BRASIL. Paraná: Pontal do Paraná, rio Guaraguaçu, 16/IV/2003, fitoplâncton, P. Bigunas, T. Ludwig \& A. Campos s.n. (UPCB47493, 47495, 47497, 47499); perifíton (UPCB47494, 47496, 47500, 47506); Matinhos, 24/X/2003, fitoplâncton, P. Bigunas, T. Ludwig \& J. Silva s.n. (UPCB47509); perifíton (UPCB47510, 47511); Pontal do Paraná, 24/X/2003, fitoplâncton, P. Bigunas, T. Ludwig \& J. Silva s.n. (UPCB47514, 47516, 47525); perifíton (UPCB47517, 47518).

14. Eunotia herzogii Krasske, Sven. Bot. Tidskr. 42(4): 426, pl. 1, fig. 20. 1948.

Fig. 24

Valvas com margem dorsal convexa apresentando quatro ondulações, depressão mediana mais pronunciada que as demais; margem ventral sutilmente côncava; extremidades subcapitadas, levemente fletidas para o lado ventral da valva, destacadas do corpo valvar, mais estreitas que a largura mediana da valva; estrias paralelas na região mediana a radiadas em direção às extremidades; aréolas inconspícuas. Compr. 24,5-27,7 $\mu \mathrm{m}$; larg. 3,2-4,7 $\mu \mathrm{m} ; 16-17$ estrias em $10 \mu \mathrm{m}$.

Distribuição geográfica para o Estado do Paraná: primeira citação de ocorrência do táxon.

Ocorrência nas amostras: BRASIL. Paraná: Pontal do Paraná, rio Guaraguaçu, 16/IV/2003, perifíton, P. Bigunas, T. Ludwig \& A. Campos s.n. (UPCB47496); 24/X/2003, fitoplâncton, P. Bigunas, T. Ludwig \& J. Silva s.n. (UPCB47512, 47516).

15. Eunotia incisa Gregory, Quart. Jour. Micr. Sci. 2: 96, pl. 4, fig. 4. 1854.

Fig. 25

Compr. 26,9-30,8 $\mu \mathrm{m}$; larg. 3,2-4 $\mu \mathrm{m} ; 12-16$ estrias em $10 \mu \mathrm{m}$.

Distribuição geográfica para o Estado do Paraná: Ponta Grossa (Fürstenberger \& Valente-Moreira 2000).

Ocorrência nas amostras: BRASIL. Paraná: Pontal do Paraná, rio Guaraguaçu, 16/IV/2003, perifíton, P. Bigunas, T. Ludwig \& A. Campos s.n. (UPCB47496); fitoplâncton (UPCB47497); Matinhos, 24/X/2003, fitoplâncton, P. Bigunas, T. Ludwig \& J. Silva s.n. (UPCB47509); Pontal do Paraná, 24/X/2003, fitoplâncton, P. Bigunas, T. Ludwig \& J. Silva s.n. (UPCB47523).

16. Eunotia indica Grunow in Rabenhorst, Beitr. Nahr. Kenntn. Verbr. Alg. 2: 5, pl. 1, fig. 7. 1865.

Fig. 26

Compr. 68,8-76,1 $\mu \mathrm{m}$; larg. 8,9-9,5 $\mu \mathrm{m} ; 16-20$ estrias em $10 \mu \mathrm{m} ; 22-24$ aréolas em $10 \mu \mathrm{m}$.

Distribuição geográfica para o Estado do Paraná: Londrina (Bittencourt-Oliveira 2002), Palmeira (ValenteMoreira 1975), Paranaguá (Moreira Filho \& ValenteMoreira 1979; Moreira Filho \& Valente-Moreira 1984) e Telêmaco Borba (Bittencourt-Oliveira 2002).

Ocorrência nas amostras: BRASIL. Paraná: Pontal do Paraná, rio Guaraguaçu, 16/IV/2003, fitoplâncton, P. Bigunas, T. Ludwig \& A. Campos s.n. (UPCB4749); 24/X/2003, perifíton, P. Bigunas, T. Ludwig \& J. Silva s.n. (UPCB47513).

17. Eunotia intermedia (Krasske ex Hustedt) Nörpel \& Lange-Bertalot in Pascher, Süss.-Fl. Mitteleur. 2(3): 215, pl. 143, fig. 10-15. 1991.

Eunotia pectinalis (Dillwyn \& Kützing) Rabenhorst var. minor (Kützing) Rabenhorst f. intermedia Krasske ex Hustedt, Akad. Verl. 7(2): 298, fig. 763. 1932.

Fig. 27-28

Compr. 9,5-16,6 $\mu \mathrm{m}$; larg. 2,4-4 $\mu \mathrm{m}$; $12-16$ estrias em $10 \mu \mathrm{m}$. 
Distribuição geográfica para o Estado do Paraná: Cascavel (Tavares \& Valente-Moreira 2000), Mangueirinha, Palmas e Pinhão (Ludwig \& Flôres 1995).

Ocorrência nas amostras: BRASIL. Paraná: Pontal do Paraná, rio Guaraguaçu, 16/IV/2003, perifíton, P. Bigunas, T. Ludwig \& A. Campos s.n. (UPCB47494, 47496, 47498, 47500, 47503, 47506); fitoplâncton (UPCB 47497); Matinhos, 24/X/2003, perifíton, P. Bigunas, T. Ludwig \& J. Silva s.n. (UPCB47510, 47511); Pontal do Paraná, 24/X/2003, fitoplâncton, P. Bigunas, T. Ludwig \& J. Silva s.n. (UPCB47512, 47514, 47516, 47519, 47521, 47523, 47524, 47525, 47526); perifíton (UPCB47513, 47515, 47517, 47518, 47520).

18. Eunotia luna var. globosa Hustedt in A. Schmidt, Atl. Diatom. pl. 286, fig. 34. 1913.

Fig. 29-30

Compr. 20,5 $\mu \mathrm{m}$; larg.: 6,9-7,9 $\mu \mathrm{m} ; 10-14$ estrias em $10 \mu \mathrm{m}$.

Distribuição geográfica para o Estado do Paraná: Curitiba (Ludwig \& Valente-Moreira 1989).

Ocorrência nas amostras: BRASIL. Paraná: Matinhos, rio Guaraguaçu, 24/X/2003, perifíton, P. Bigunas, T. Ludwig \& J. Silva s.n. (UPCB47511).

19. Eunotia maior (Wm. Smith) Rabenhorst, Eur. Alg., sect. 1, p. 72. 1864.

Himantidium majus Wm. Smith, British. Diat. 2: 14, pl. 33, fig. 286. 1856.

Fig. 31-32

Compr. 41,3-68,2 $\mu \mathrm{m}$; larg. 8,2-10,6 $\mu \mathrm{m} ; 8-11$ estrias em $10 \mu \mathrm{m} ; 18-20$ aréolas em $10 \mu \mathrm{m}$.

Distribuição geográfica para o Estado do Paraná: Caiobá (Valente-Moreira \& Moreira Filho 1982), Curitiba (Ludwig \& Valente-Moreira 1989), Ponta Grossa (Moro et al. 1994).

Ocorrência nas amostras: BRASIL. Paraná: Pontal do Paraná, rio Guaraguaçu, 24/X/2003, fitoplâncton, P. Bigunas, T. Ludwig \& J. Silva s.n. (UPCB47509); perifíton (UPCB47510).

20. Eunotia meridiana Metzeltin \& Lange-Bertalot, Iconogr. Diatomol. 5: 67, pl. 59, fig. 7-10. 1998.

Fig. 33-35

Valvas com margem dorsal convexa; margem ventral reta a côncava apresentando pequena convexidade próxima às extremidades; extremidades arredondadas a atenuado-arredondadas, não destacadas do corpo valvar; estrias paralelas a radiadas em direção às extremidades; aréolas delicadas, geralmente inconspícuas. Compr. 15-33,2 $\mu \mathrm{m}$; larg. 4,7-8,7 $\mu \mathrm{m} ; 12-14$ estrias em $10 \mu \mathrm{m}$; 20-24 aréolas em $10 \mu \mathrm{m}$.
Os indivíduos analisados assemelham-se a Eunotia pirla Carter \& Flower, mas esta última apresenta maior concavidade na margem ventral, extremidades mais destacadas do corpo valvar, número levemente mais elevado de estrias (cerca de $17 \mathrm{em} 10 \mu \mathrm{m}$ ) e maior densidade de estrias nas extremidades da valva (Carter $\&$ Flower 1988).

Distribuição geográfica para o Estado do Paraná: primeira citação de ocorrência do táxon.

Ocorrência nas amostras: BRASIL. Paraná: Pontal do Paraná, rio Guaraguaçu, 16/IV/2003, perifíton, P. Bigunas, T. Ludwig \& A. Campos s.n. (UPCB47502, 47503); Matinhos, 24/X/2003, perifíton, P. Bigunas, T. Ludwig \& J. Silva s.n. (UPCB47510, 47511); Pontal do Paraná, 24/X/2003, fitoplâncton, P. Bigunas, T. Ludwig \& J. Silva s.n. (UPCB47512, 47514, 47516, 47519, 47523, 47524, 47525, 47521); perifíton (UPCB47513, 47520).

21. Eunotia minor (Kützing) Grunow in Van Heurck, Syn. Diat. Belg. pl. 33, fig. 20-21. 1881.

Himantidium minus Kützing, Bacill. p. 39, pl. 16, fig. 10. 1844.

Fig. 36

Compr. 18,1-21,3 $\mu \mathrm{m}$, larg. 3,9-5 $\mu \mathrm{m}, 12-14$ estrias em $10 \mu \mathrm{m}$.

Distribuição geográfica para o Estado do Paraná: Curitiba (Moreira-Filho et al. 1973; Ludwig \& ValenteMoreira 1989; Contin 1990, Ludwig et al. 2005), Mangueirinha, Palmas e Pinhão (Ludwig \& Flôres 1995), Ponta Grossa (Moreira Filho et al. 1976; Moro et al. 1994).

Ocorrência nas amostras: BRASIL. Paraná: Pontal do Paraná, rio Guaraguaçu, 24/X/2003, fitoplâncton, P. Bigunas, T. Ludwig \& J. Silva s.n. (UPCB47512, 47516); perifíton (UPCB47518).

22. Eunotia monodon Ehrenberg, Abh. Akad. Wiss. Berl. p. 414, pl. 2, fig. 7. 1841 (1843).

Fig. 37

Compr. 35,4-78,6 $\mu \mathrm{m}$; larg. 7,5-9,6 $\mu \mathrm{m} ; 8$-12 estrias em $10 \mu \mathrm{m}$; 16-24 aréolas em $10 \mu \mathrm{m}$.

Distribuição geográfica para o Estado do Paraná: Caiobá (Valente-Moreira \& Moreira Filho 1982), Curitiba (Moreira Filho \& Momoli 1966; Moreira Filho et al. 1973; Lozovei \& Luz 1976; Contin 1990; Ludwig \& ValenteMoreira 1989; Lozovei \& Shirata 1990), Guaratuba (Moreira Filho 1961; Moreira Filho \& Valente-Moreira 1984), Ipiranga e Londrina (Bittencourt-Oliveira 2002), Mangueirinha (Ludwig \& Flôres 1995), Palmeira (Valente-Moreira 1975), Paranaguá (Valente-Moreira et al. 1986), Pinhão (Ludwig \& Flôres 1995), Ponta Grossa (Fürstenberger \& Valente-Moreira 2000), Sapopema 
(Bittencourt-Oliveira 2002), São José dos Pinhais (Momoli 1967), Sertanópolis e Telêmaco Borba (Bittencourt-Oliveira 2002).

Ocorrência nas amostras: BRASIL. Paraná: Pontal do Paraná, rio Guaraguaçu, 16/IV/2003, fitoplâncton,
P. Bigunas, T. Ludwig \& A. Campos s.n. (UPCB47493, 47497, 47499); perifíton (UPCB47494, 47496, 47498, 47500); Matinhos, 24/X/2003, fitoplâncton, P. Bigunas, T. Ludwig \& J. Silva s.n. (UPCB 47509); perifíton (UPCB 47511); Pontal do Paraná, 24/X/2003, fitoplâncton,
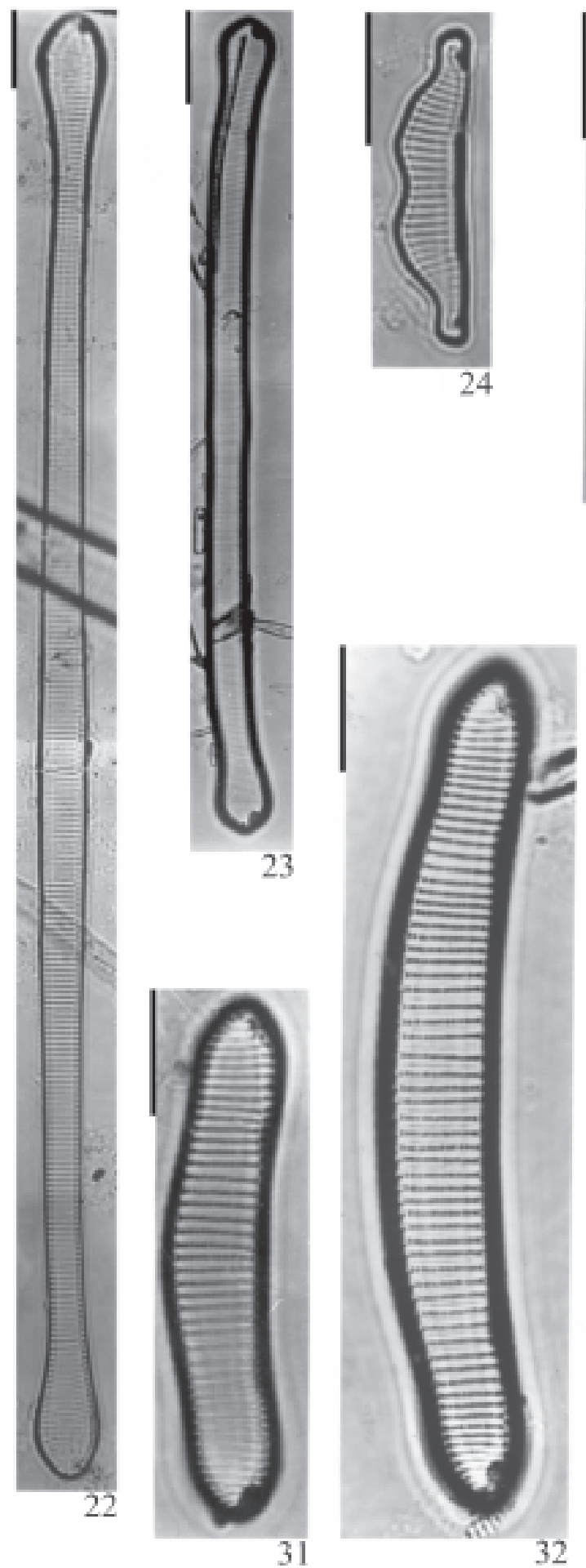
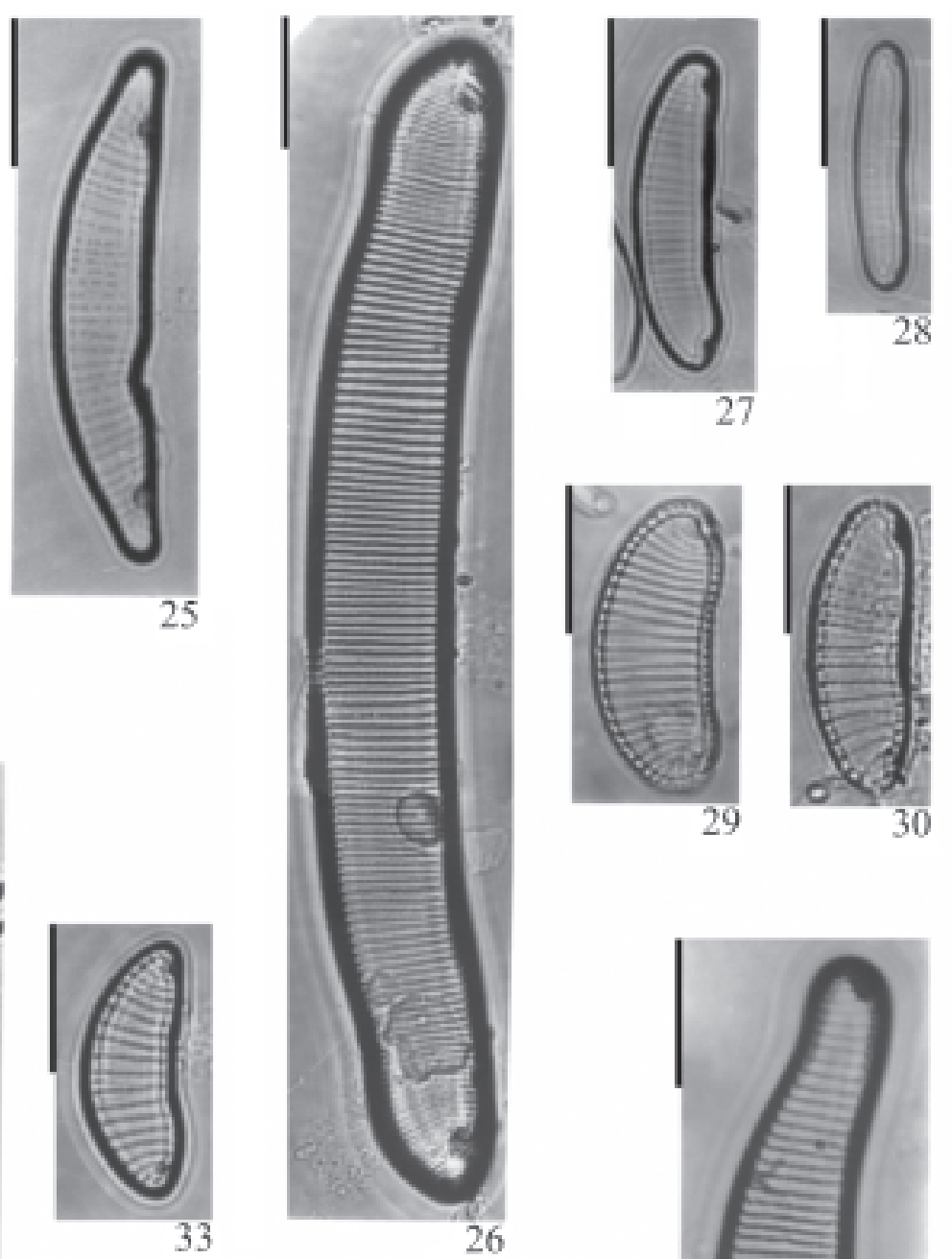

30
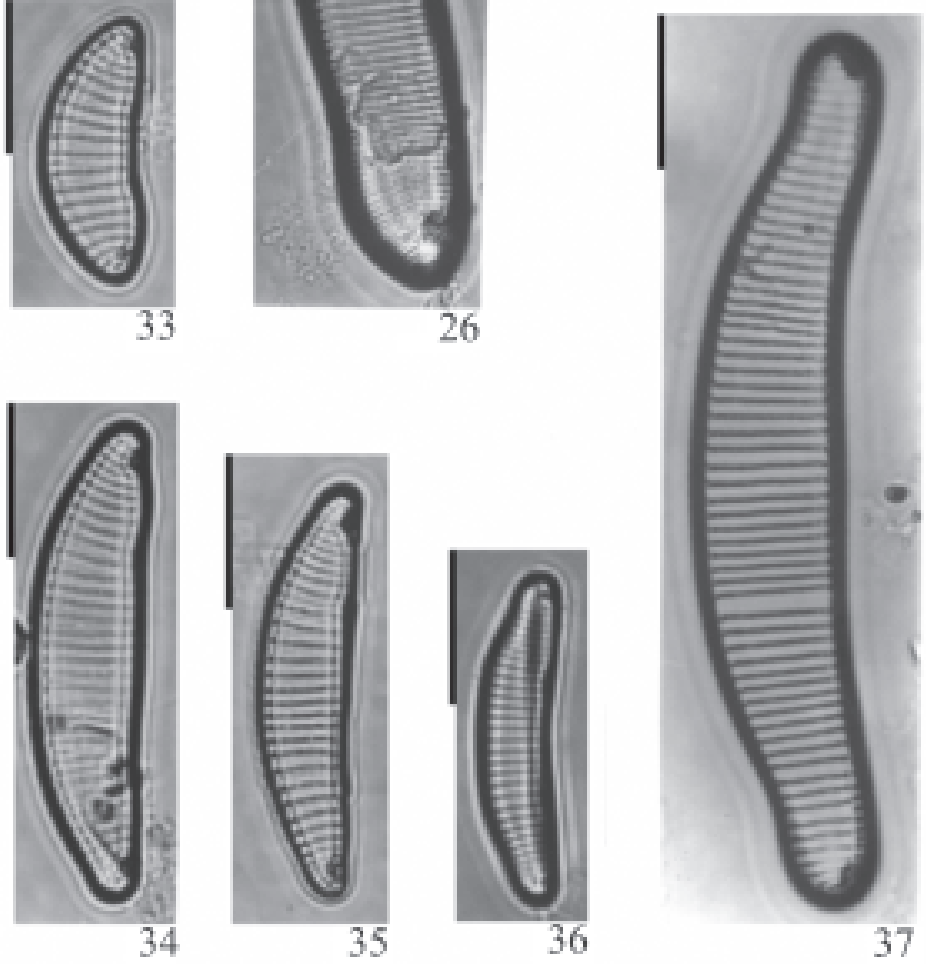

Figuras 22-37. Eunotia Ehrenberg (Bacillariophyceae) do rio Guaraguaçu, Paraná, Brasil. 22. Eunotia flexuosa Brébisson ex Kützing. 23. Eunotia formica Ehrenberg. 24. Eunotia herzogii Krasske. 25. Eunotia incisa Gregory. 26. Eunotia indica Grunow. 27-28. Eunotia intermedia (Krasske ex Hustedt) Nörpel \& Lange-Bertalot. 29-30. Eunotia luna var. globosa Hustedt. 31-32. Eunotia maior (Wm. Smith) Rabenhorst. 33-35. Eunotia meridiana Metzeltin \& Lange-Bertalot. 36. Eunotia minor (Kützing) Grunow. 37. Eunotia monodon Ehrenberg. Barras $=10 \mu \mathrm{m}$. 
P. Bigunas, T. Ludwig \& J. Silva s.n. (UPCB47512, 47514, 47523, 47524); perifíton (UPCB47513, 47515, 47518).

23. Eunotia muscicola var. tridentula Nörpel \& LangeBertalot in Pascher, Süss.-Fl. Mitteur. 2: 3, pl. 156, fig. 12-22. 1991.

Fig. 38-39

Valvas com margem dorsal convexa apresentando três ondulações; margem ventral côncava; extremidades capitadas a subcapitadas, destacadas do corpo valvar, mais estreitas que a largura mediana da valva; estrias paralelas medianamente a radiadas em direção às extremidades; aréolas inconspícuas. Compr.: 14,2-15 $\mu \mathrm{m}$; larg. 3,2-4 $\mu \mathrm{m} ; 16-20$ estrias em $10 \mu \mathrm{m}$.

Apesar da variedade típica da espécie ser mais comum na América do Sul, esta apresenta uma curvatura menos pronunciada na margem ventral da valva do que a variedade tridentula. Além disso, as duas variedades apresentam pequena diferença nos valores de eixo transapical. A var. muscicola apresenta em média $3 \mu \mathrm{m}$ de largura e a var. tridentula, 3,5 $\mu \mathrm{m}$ (Krammer \& Lange-Bertalot 1991).

Distribuição geográfica para o Estado do Paraná: primeira citação de ocorrência do táxon.

Ocorrência nas amostras: BRASIL. Paraná: Pontal do Paraná, rio Guaraguaçu, 16/IV/2003, perifíton, P. Bigunas, T. Ludwig \& A. Campos s.n. (UPCB47500); Pontal do Paraná, 24/X/2003, fitoplâncton, P. Bigunas, T. Ludwig \& J. Silva s.n. (UPCB47512, 47525).

24. Eunotia nymanniana Grunow in Van Heurck, Syn. Diat. Belg. pl. 34, fig. 8. 1881.

Fig. 40-42

Valvas com margem dorsal levemente convexa; margem ventral levemente côncava; extremidades capitadas, fletidas dorsalmente, destacadas do corpo valvar, mais estreitas que a largura mediana da valva; estrias paralelas a radiadas em direção às extremidades; aréolas inconspícuas. Compr. 15,8-23,7 $\mu \mathrm{m}$; larg. 2,5-3,2 $\mu \mathrm{m}$; 16-18 estrias em $10 \mu \mathrm{m}$.

Distribuição geográfica para o Estado do Paraná: primeira citação de ocorrência do táxon.

Ocorrência nas amostras: BRASIL. Paraná: Pontal do Paraná, rio Guaraguaçu, 16/IV/2003, fitoplâncton, P. Bigunas, T. Ludwig \& A. Campos s.n. (UPCB47499, 47501); Matinhos, 24/X/2003, perifíton, P. Bigunas, T. Ludwig \& J. Silva s.n. (UPCB47511); Pontal do Paraná, 24/X/2003, fitoplâncton, P. Bigunas, T. Ludwig \& J. Silva s.n. (UPCB47514, 47516, 47521, 47524); perifíton (UPCB47515, 47518).

25. Eunotia praerupta Ehrenberg var. praerupta, Phys. Akad. Wiss. Berl. p. 414.1841 (1843).

Fig. 43
Compr. 41,9-79 $\mu \mathrm{m}$; larg. 12,3-15,8 $\mu \mathrm{m} ; 8-14$ estrias em $10 \mu \mathrm{m} ; 20$ aréolas em $10 \mu \mathrm{m}$.

Distribuição geográfica para o Estado do Paraná: Curitiba (Moreira Filho \& Momoli 1966; Moreira Filho et al. 1973; Lozovei \& Shirata 1990), Ponta Grossa (Moreira Filho et al. 1976), Sapopema (BittencourtOliveira 2002), São José dos Pinhais (Momoli 1967).

Ocorrência nas amostras: BRASIL. Paraná: Pontal do Paraná, rio Guaraguaçu, 16/IV/2003, fitoplâncton, P. Bigunas, T. Ludwig \& A. Campos s.n. (UPCB47493, 47495, 47497); perifíton (UPCB47496); Matinhos, 24/X/2003, fitoplâncton, P. Bigunas, T. Ludwig \& J. Silva s.n. (UPCB47509); Pontal do Paraná, 24/X/2003, perifíton, P. Bigunas, T. Ludwig \& J. Silva s.n. (UPCB47513, 47515).

26. Eunotia praerupta var. bidens (Ehrenberg) Grunow in Cleve \& Grunow, Sven. Vet. Akad. Handl. 17(2): 109. 1880.

Eunotia bidens Ehrenberg, Abh. Akad. Wiss. Berl., p. 413. 1841 (1843).

Fig. 44-45

Compr. 35,6-65,6 $\mu \mathrm{m}$; larg. 7,9-15,8 $\mu \mathrm{m} ; 10-13$ estrias em $10 \mu \mathrm{m}$.

Distribuição geográfica para o Estado do Paraná: Curitiba (Moreira Filho \& Momoli 1966; Moreira Filho et al. 1973; Ludwig \& Valente-Moreira 1989; Contin 1990), Mangueirinha e Pinhão (Ludwig \& Flôres 1995), Sapopema e Telêmaco Borba (Bittencourt-Oliveira 2002).

Ocorrência nas amostras: BRASIL. Paraná: Pontal do Paraná, rio Guaraguaçu, 16/IV/2003, perifíton, P. Bigunas, T. Ludwig \& A. Campos s.n. (UPCB47496); Matinhos, 24/X/2003, fitoplâncton, P. Bigunas, T. Ludwig \& J. Silva s.n. (UPCB47509); perifíton (UPCB47510, 47511); Pontal do Paraná, 24/X/2003, fitoplâncton, P. Bigunas, T. Ludwig \& J. Silva s.n. (UPCB47512, 47514, 47516, 47524, 47525); perifíton (UPCB47513, 47515, 47517, 47518, 47520).

27. Eunotia pseudoindica Frenguelli, Rev. Mus. La Plata, Bot. 3: 307. 1941.

Fig. 46

Valvas com margem dorsal convexa; margem ventral côncava; extremidades cuneadas, destacadas do corpo valvar, mais estreitas que a largura mediana da valva; estrias paralelas a radiadas em direção às extremidades; aréolas delicadas. Compr.: 42,8-59,4 $\mu \mathrm{m}$; larg. 7,9-9,3 $\mu \mathrm{m} ; 14-18$ estrias em $10 \mu \mathrm{m} ; 22-26$ aréolas em $10 \mu \mathrm{m}$.

Frenguelli (1941) propõe a espécie E. pseudoindica com base em exemplares anteriormente descritos por ele como $E$. indica. Estes exemplares foram encontrados em amostras provenientes de um sistema de banhados e 
lagoas de Yberá, na Argentina. A diferença entre E. pseudoindica e E. indica baseia-se principalmente na forma das extremidades valvares. E. indica apresenta extremidades cuneado-arredondadas e E. pseudoindica extremidades cuneadas, mais atenuadas e alongadas. Além disso, o autor comenta que $E$. indica está mais relacionada com o grupo de E. monodon e que E. pseudoindica assemelha-se mais com as formas de E. zygodon, principalmente no que se refere ao tamanho e à forma da margem dorsal da valva.

Distribuição geográfica para o Estado do Paraná: primeira citação de ocorrência do táxon.

Ocorrência nas amostras: BRASIL. Paraná: Pontal do Paraná, rio Guaraguaçu, 16/IV/2003, perifíton, P. Bigunas, T. Ludwig \& A. Campos s.n. (UPCB47494); fitoplâncton (UPCB47495, 47497); Pontal do Paraná, 24/X/2003, perifíton, P. Bigunas, T. Ludwig \& J. Silva s.n. (UPCB47513, 47515, 47517); fitoplâncton (UPCB47516, 47525).

28. Eunotia pyramidata Hustedt var. pyramidata in A. Schmidt, Atl. Diatom. pl. 286, fig. 20-24. 1913. Fig. 47-50

Compr. 22,9-42,1 $\mu \mathrm{m}$; larg. 7,1-8,7 $\mu \mathrm{m} ; 10-13$ estrias em $10 \mu \mathrm{m}$.

Distribuição geográfica para o Estado do Paraná: Curitiba (Moreira Filho et al. 1973; Ludwig \& ValenteMoreira 1989; Contin 1990; Lozovei \& Shirata 1990; Ludwig et al. 2005), Pinhão (Ludwig \& Flôres 1995).

Ocorrência nas amostras: BRASIL. Paraná: Pontal do Paraná, rio Guaraguaçu, 16/IV/2003, fitoplâncton, P. Bigunas, T. Ludwig \& A. Campos s.n. (UPCB47493, 47497); perifíton (UPCB47496); Matinhos, 24/X/2003, fitoplâncton, P. Bigunas, T. Ludwig \& J. Silva s.n. (UPCB47509); perifíton (UPCB47511); Pontal do Paraná, 24/X/2003, fitoplâncton, P. Bigunas, T. Ludwig \& J. Silva s.n. (UPCB47516, 47523, 47524, 47525).

29. Eunotia pyramidata var. monodon Krasske, Arch. Hydrobiol. 35: 365, pl. 10, fig. 26-28, 35, 44. 1939. Fig. 51

Compr. 17,5 $\mu \mathrm{m}$; larg. 6,9 $\mu \mathrm{m}$; 16 estrias em $10 \mu \mathrm{m}$. Distribuição geográfica para o Estado do Paraná: Curitiba (Ludwig \& Valente-Moreira 1989; Contin 1990).

Ocorrência nas amostras: BRASIL. Paraná: Pontal do Paraná, rio Guaraguaçu, 16/IV/2003, perifíton, P. Bigunas, T. Ludwig \& A. Campos s.n. (UPCB47496).

30. Eunotia rabenhorstiana (Grunow) Hustedt, Süssw. Diat. Albert. Nat. Park. p. 72. 1949.

Desmogonium rabenhorstianum Grunow in Rabenhorst, Beitr., 1: 6, pl. 1, fig. 1, 1865.

Fig. 52
Compr. 67,9-146,9 $\mu \mathrm{m}$; larg. 5,5-8,7 $\mu \mathrm{m} ; 12-20$ estrias em $10 \mu \mathrm{m}$.

Distribuição geográfica para o Estado do Paraná: Curitiba (Moreira Filho et al. 1973; Ludwig et al. 2005), Pontal do Paraná (Valente-Moreira et al. 1994).

Ocorrência nas amostras: BRASIL. Paraná: Pontal do Paraná, rio Guaraguaçu, 16/IV/2003, perifíton, P. Bigunas, T. Ludwig \& A. Campos s.n. (UPCB47494, 47498, 47503); Matinhos, 24/X/2003, perifíton, P. Bigunas, T. Ludwig \& J. Silva s.n. (UPCB47511); Pontal do Paraná, 24/X/2003, fitoplâncton, P. Bigunas, T. Ludwig \& J. Silva s.n. (UPCB47512, 47525); perifíton (UPCB47513, 47518).

31. Eunotia rabenhorstii Cleve \& Grunow in Van Heurck, Syn. Diat. Belg. pl. 35, fig. 12. 1881.

Fig. 53-55

Compr. 15,8-30 $\mu \mathrm{m}$; larg. 5,5-7,9 $\mu \mathrm{m} ; 10-15$ estrias em $10 \mu \mathrm{m}$.

Distribuição geográfica para o Estado do Paraná: Curitiba (Moreira Filho \& Momoli 1966; Moreira Filho et al. 1973; Ludwig \& Valente-Moreira 1989; Contin 1990; Lozovei \& Shirata 1990; Ludwig et al. 2005), Mangueirinha e Palmas (Ludwig \& Flôres 1995), Paranaguá (Valente-Moreira et al. 1986), Pinhão (Ludwig \& Flôres 1995), Ponta Grossa (Fürstenberger \& ValenteMoreira 2000; Moro et al. 1994), São José dos Pinhais (Momoli 1967).

Ocorrência nas amostras: BRASIL. Paraná: Pontal do Paraná, rio Guaraguaçu, 16/IV/2003, fitoplâncton, $P$. Bigunas, T. Ludwig \& A. Campos s.n. (UPCB47493, 47495, 47497, 47499); perifíton (UPCB47494, 47496, 47498, 47500); Matinhos, 24/X/2003, fitoplâncton, P. Bigunas, T. Ludwig \& J. Silva s.n. (UPCB47509); perifíton (UPCB47510, 47511); Pontal do Paraná, 24/X/2003, fitoplâncton, P. Bigunas, T. Ludwig \& J. Silva s.n. (UPCB47512, 47514, 47516, 47519, 47521, 47523, 47524, 47525); perifíton (UPCB47513, 47515, 47517, 47518, 47520).

32. Eunotia schneideri Metzeltin \& Lange-Bertalot, Iconogr. Diatomol. 5: 77, fig. 1-2. 1998.

Fig. 56

Compr. 20,5-25,3 $\mu \mathrm{m}$; larg. 3,2-4,7 $\mu \mathrm{m} ; 12-14$ estrias em $10 \mu \mathrm{m}$.

E. schneideri e E. bidentula W. Smith são espécies muito próximas que se distinguem pelo habitat, densidade de estrias e largura das ondulações. E. schneideri ocorre em regiões quentes, apresenta cerca de 12 estrias em $10 \mu \mathrm{m}$ e ondulações delgadas, pouco pronunciadas. Já E. bidentula, ocorre em locais mais frios, possui 15-20 estrias em $10 \mu \mathrm{m}$ e ondulações largas, pronunciadas (Metzeltin \& Lange-Bertalot 1998; Krammer \& LangeBertalot 1991). 
Distribuição geográfica para o Estado do Paraná: Curitiba (Contin 1990 - citada como Eunotia bidentula).

Ocorrência nas amostras: BRASIL. Paraná: Pontal do Paraná, rio Guaraguaçu, 16/IV/2003, fitoplâncton,
P. Bigunas, T. Ludwig \& A. Campos s.n. (UPCB47495, 47497, 47499); Pontal do Paraná, 24/X/2003, fitoplâncton, P. Bigunas, T. Ludwig \& J. Silva s.n. (UPCB47512, 47519, 47523); perifíton (UPCB47513, 47515).
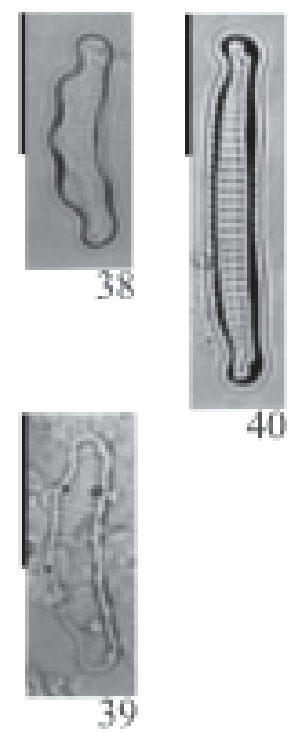

40
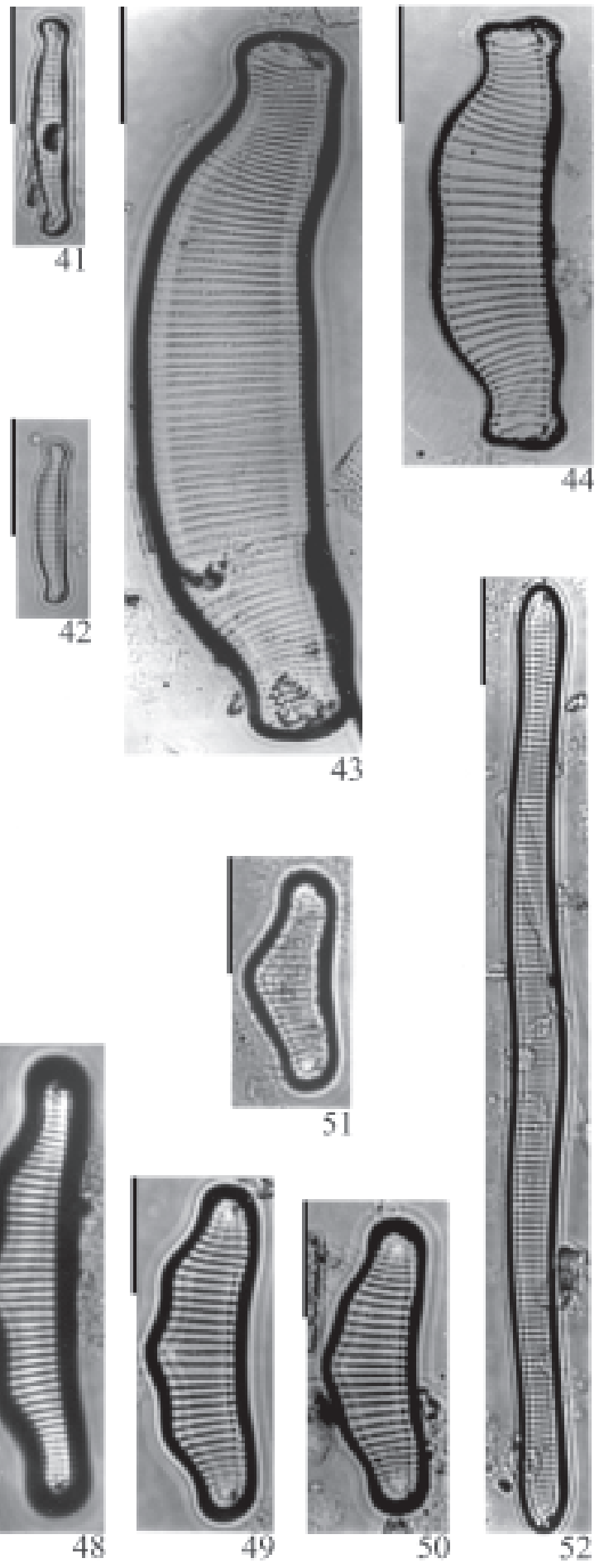

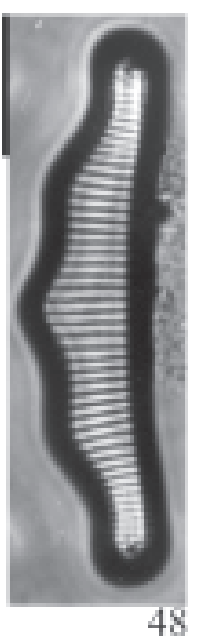

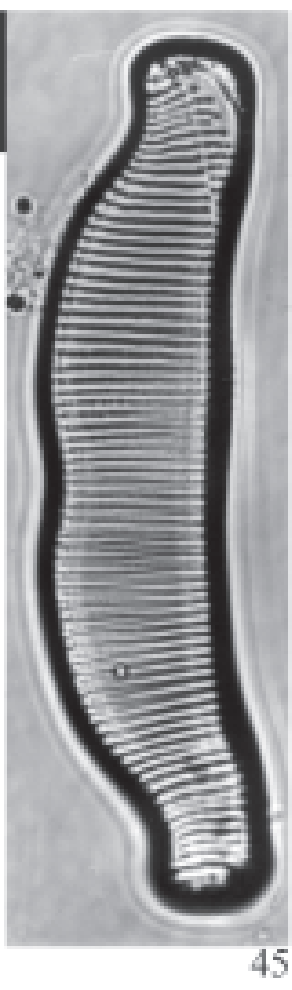
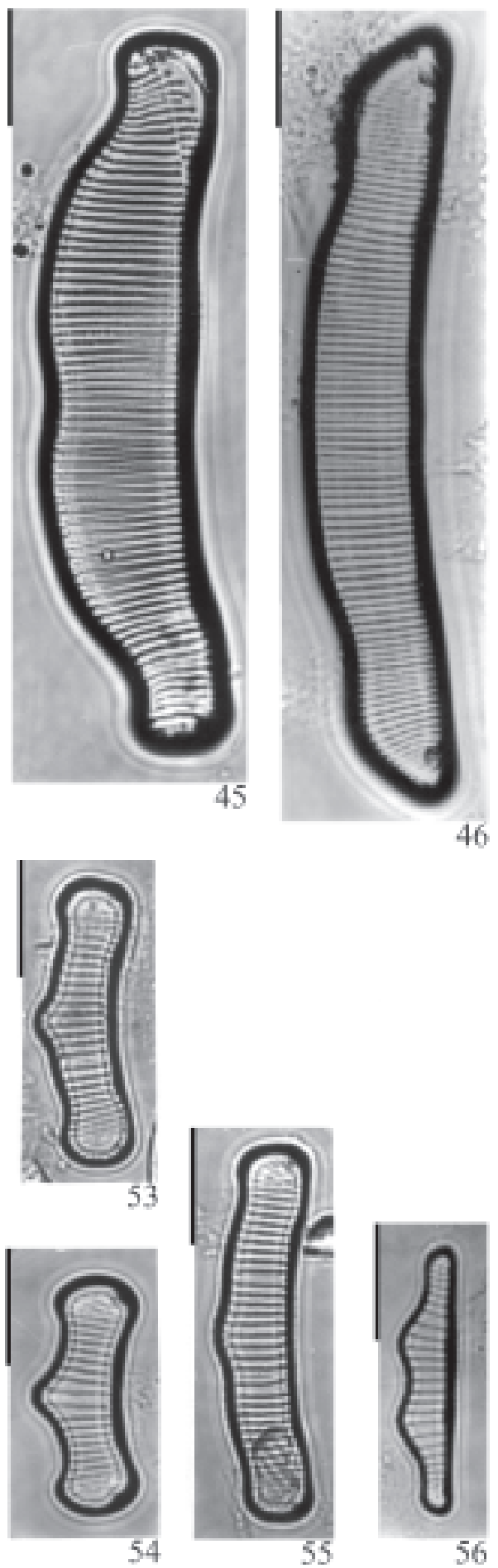

Figuras 38-56. Eunotia Ehrenberg (Bacillariophyceae) do rio Guaraguaçu, Paraná, Brasil. 38-39. Eunotia muscicola var. tridentula Nörpel \& Lange-Bertalot. 40-42. Eunotia nymanniana Grunow. 43. Eunotia praerupta Ehrenberg var. praerupta. 44-45. Eunotia praerupta var. bidens (Ehrenberg) Grunow. 46. Eunotia pseudoindica Frenguelli. 47-50. Eunotia pyramidata Hustedt var. pyramidata. 51. Eunotia pyramidata var. monodon Krasske. 52. Eunotia rabenhorstiana (Grunow) Hustedt. 53-55. Eunotia rabenhorstii Cleve \& Grunow. 56. Eunotia schneideri Metzeltin \& Lange-Bertalot. Barras $=10 \mu \mathrm{m}$. 
33. Eunotia serra Ehrenberg, Ber. Akad. Wiss. Berl. p. 45.1837.

Fig. 57

Compr. 56,7-71,1 $\mu \mathrm{m}$; larg. 8,7-11,9 $\mu \mathrm{m} ; 11-12$ estrias em $10 \mu \mathrm{m}$.

Distribuição geográfica para o Estado do Paraná: Palmas (Ludwig \& Flôres 1995), Telêmaco Borba (Bittencourt-Oliveira 2002).

Ocorrência nas amostras: BRASIL. Paraná: Pontal do Paraná, rio Guaraguaçu, 16/IV/2003, perifíton, P. Bigunas, T. Ludwig \& A. Campos s.n. (UPCB47496, 47506); Matinhos, 24/X/2003, perifíton, P. Bigunas, T. Ludwig \& J. Silva s.n. (UPCB47511); Pontal do Paraná, 24/X/2003, perifíton, P. Bigunas, T. Ludwig \& J. Silva s.n. (UPCB47513); fitoplâncton (UPCB47514).

34. Eunotia sudetica O. Müller, Forschungsber. Biol. Stat. Plön. 6: 12, pl. 3, fig. 25-26. 1898.

Fig. 58-59

Compr. 19,8-30,2 $\mu \mathrm{m}$; larg. 4,7-7,1 $\mu \mathrm{m} ; 11-15$ estrias em $10 \mu \mathrm{m}$.

Distribuição geográfica para o Estado do Paraná: Caiobá (Valente-Moreira \& Moreira Filho 1982), Cascavel (Tavares \& Valente-Moreira 2000), Curitiba (Moreira Filho et al. 1973; Ludwig \& Valente-Moreira 1989; Contin 1990), Mangueirinha e Palmas (Ludwig \& Flôres 1995), Palmeira (Valente-Moreira 1975), Paranaguá (Moreira Filho \& Valente-Moreira 1979), Pinhão (Ludwig \& Flôres 1995), Ponta Grossa (Moreira Filho et al. 1976; Fürstenberger \& Valente-Moreira 2000), Pontal do Paraná (Valente-Moreira \& Moreira Filho 1981).

Ocorrência nas amostras: BRASIL. Paraná: Pontal do Paraná, rio Guaraguaçu, 16/IV/2003, fitoplâncton, P. Bigunas, T. Ludwig \& A. Campos s.n. (UPCB47501); perifíton (UPCB47502); Matinhos, 24/X/2003, perifíton, P. Bigunas, T. Ludwig \& J. Silva s.n. (UPCB47510, 47511); Pontal do Paraná, 24/X/2003, fitoplâncton, P. Bigunas, T. Ludwig \& J. Silva s.n. (UPCB47512, 47514, 47516, 47519, 47523, 47525, 47526); perifíton (UPCB47513, 47515, 47517, 47518).

35. Eunotia transfuga Metzeltin \& Lange-Bertalot, Iconogr. Diatomol. 5: 84, pl. 8, fig. 5, pl. 9, fig. 1-3. 1998.

Fig. 60

Compr. 98-181,7 $\mu \mathrm{m}$; larg. 7,9-9,5 $\mu \mathrm{m} ; 118$ estrias em $10 \mu \mathrm{m} ; 14$ aréolas em $10 \mu \mathrm{m}$.

Distribuição geográfica para o Estado do Paraná: Palmas (Ludwig \& Flôres 1995 - citada como Eunotia lineolata Hustedt).

Ocorrência nas amostras: BRASIL. Paraná: Pontal do Paraná, rio Guaraguaçu, 16/IV/2003, fitoplâncton, $P$. Bigunas, T. Ludwig \& A. Campos s.n. (UPCB47493, 47495, 47497, 47499); perifíton (UPCB47494, 47498,
47500, 47502, 47503, 47506); Matinhos, 24/X/2003, fitoplâncton, P. Bigunas, T. Ludwig \& J. Silva s.n. (UPCB47509); perifíton (UPCB47510, 47511); Pontal do Paraná, 24/X/2003, fitoplâncton, P. Bigunas, T. Ludwig \& J. Silva s.n. (UPCB47512, 47514, 47516, 47523, 47524, 47525); perifíton (UPCB47513, 47515, 47518, 47520).

36. Eunotia trigibba Hustedt in A. Schmidt, Atl. Diatom. pl. 286, fig. 16-18. 1913.

Fig. 61

Compr. 24,5-40,3 $\mu \mathrm{m}$; larg. 8,7-11,1 $\mu \mathrm{m} ; 9-18$ estrias em $10 \mu \mathrm{m} ; 24$ ou mais aréolas em $10 \mu \mathrm{m}$.

Distribuição geográfica para o Estado do Paraná: Curitiba (Ludwig \& Valente-Moreira 1989; Contin 1990).

Ocorrência nas amostras: BRASIL. Paraná: Pontal do Paraná, rio Guaraguaçu, 16/IV/2003, perifíton, P. Bigunas, T. Ludwig \& A. Campos s.n. (UPCB47494, 47500, 47506); fitoplâncton (UPCB47495, 47497, 47499); Matinhos, 24/X/2003, fitoplâncton, P. Bigunas, T. Ludwig \& J. Silva s.n. (UPCB47509); perifíton (UPCB47510); Pontal do Paraná, 24/X/2003, perifíton, P. Bigunas, T. Ludwig \& J. Silva s.n. (UPCB47515, 47520); fitoplâncton (UPCB47516, 47523, 47524, 47525).

37. Eunotia trinacria Krasske, Beitr. Diat. Sach. Bot. Arch. p. 349, fig. a-d. 1929.

Fig. 62-63

Valvas com margem dorsal convexa apresentando três ondulações, ondulação mediana um pouco mais proeminente que as demais; margem ventral reta a sutilmente côncava; extremidades subcapitadas; sutilmente destacadas do corpo valvar, mais estreitas que a largura mediana da valva; estrias delicadas, paralelas a radiadas nas extremidades; aréolas inconspícuas. Compr. 16,6-23,7 $\mu \mathrm{m}$; larg. 2,4 $\mu \mathrm{m}$; 18-22 estrias em $10 \mu \mathrm{m}$.

Distribuição geográfica para o Estado do Paraná: primeira citação de ocorrência do táxon.

Ocorrência nas amostras: BRASIL. Paraná: Pontal do Paraná, rio Guaraguaçu, 16/IV/2003, perifíton, P. Bigunas, T. Ludwig \& A. Campos s.n. (UPCB47494, 47500); Matinhos, 24/X/2003, perifíton, P. Bigunas, T. Ludwig \& J. Silva s.n. (UPCB47510); Pontal do Paraná, 24/X/2003, fitoplâncton, P. Bigunas, T. Ludwig \& J. Silva s.n. (UPCB47512, 47516, 47519, 47521, 47523, 47524, 47525); perifíton (UPCB47513).

38. Eunotia veneris (Kützing) De Toni, Syll. Alg. 2(2): 794. 1892.

Himantidium veneris Kützing, Bacill. p. 40, pl. 30, fig. 7. 1844.

Fig. 64

Compr. 42,4-45,3 $\mu \mathrm{m}$; larg. 5,6-6,3 $\mu \mathrm{m} ; 15-18$ estrias em $10 \mu \mathrm{m}$. 
Distribuição geográfica para o Estado do Paraná: Cascavel (Tavares \& Valente-Moreira 2000).

Ocorrência nas amostras: UPCB BRASIL. Paraná: Pontal do Paraná, rio Guaraguaçu, 24/X/2003, fitoplâncton, P. Bigunas, T. Ludwig \& J. Silva s.n. (UPCB47514).

39. Eunotia zygodon Ehrenberg, Abh. Akad. Wiss. Berl. p. 415, pl. 2, fig. 6. 1841 (1843).

Fig. 65

Compr. 55,3-107,4 $\mu \mathrm{m}$; larg. 11,9-15,8 $\mu \mathrm{m} ; 10-12$ estrias em $10 \mu \mathrm{m} ; 20-24$ aréolas em $10 \mu \mathrm{m}$.

Distribuição geográfica para o Estado do Paraná: Curitiba (Contin 1990), Londrina (Bittencourt-Oliveira 2002), Palmeira (Valente-Moreira 1975), Ponta Grossa (Fürstenberger \& Valente-Moreira 2000), Sertanópolis e Telêmaco Borba (Bittencourt-Oliveira 2002).

Ocorrência nas amostras: BRASIL. Paraná: Pontal do Paraná, rio Guaraguaçu, 16/IV/2003, perifíton, P. Bigunas, T. Ludwig \& A. Campos s.n. (UPCB47494, 47496, 47498, 47503); fitoplâncton (UPCB47497); Matinhos, 24/X/2003, fitoplâncton, P. Bigunas, T. Ludwig \& J. Silva s.n. (UPCB47509); perifíton (UPCB47510, 47511); Pontal do Paraná, 24/X/2003, perifíton, P. Bigunas, T. Ludwig \& J. Silva s.n. (UPCB47513, 47517, 47518, 47520); fitoplâncton (UPCB47514, 47516, 47523, 47524, 47525).

\section{Eunotia sp. 1}

Fig. 66-68

Compr. 25,3-77,4 $\mu \mathrm{m}$; larg. 4-6,3 $\mu \mathrm{m} ; 12-18$ estrias em $10 \mu \mathrm{m}$.

Exemplares similares a Eunotia sp. 1 foram registrados como Eunotia faba (Ehrenberg) Grunow por Fürstenberger \& Valente-Moreira (2000) em estudo das Eunotiaceae em Ponta Grossa, Paraná. Contudo, ilustrações e descrições apresentadas para $E$. faba em Hustedt (1927-1966), Krammer \& Lange-Bertalot (1991) e Schmidt (1874-1959) caracterizam a espécie pelas extremidades arredondadas, nódulos terminais da rafe menos grosseiros e margem ventral geralmente reta, detalhes morfológicos distintos dos de Eunotia sp. 1.

Distribuição geográfica para o Estado do Paraná: Ponta Grossa (Fürstenberger \& Valente-Moreira 2000 citada como Eunotia faba).

Ocorrência nas amostras: BRASIL. Paraná: Pontal do Paraná, rio Guaraguaçu, 16/IV/2003, fitoplâncton, P. Bigunas, T. Ludwig \& A. Campos s.n. (UPCB47501); perifíton (UPCB47502, 47503); Matinhos, 24/X/2003, perifíton, P. Bigunas, T. Ludwig \& J. Silva s.n. (UPCB47510, 47511); Pontal do Paraná, 24/X/2003, fitoplâncton, P. Bigunas, T. Ludwig \& J. Silva s.n. (UPCB47512，47514，47523, 47519); perifíton (UPCB47513, 47515, 47518).

\section{Eunotia sp. 2}

Fig. 69

Valvas com margem dorsal convexa apresentando quatro ondulações, depressão mediana mais pronunciada que as demais; margem ventral côncava; extremidades capitadas, fletidas dorsalmente, destacadas do corpo valvar, mais estreitas que a largura mediana da valva; nódulos terminais nas extremidades; estrias paralelas a radiadas em direção às extremidades; aréolas inconspícuas. Compr. 26,9-36,3 $\mu \mathrm{m}$; larg. 6,3-7,5 $\mu \mathrm{m}$; 12-14 estrias em $10 \mu \mathrm{m}$.

Eunotia sp. 2 assemelha-se à exemplares de E. camelus Ehrenberg ilustrados por Metzeltin \& LangeBertalot (1998). Porém, Eunotia sp. 2 apresenta extremidades capitadas com maior flexão dorsal e ondulações mais largas do que E. camelus que possui extremidades rostrado-arredondadas.

Distribuição geográfica para o Estado do Paraná: primeira citação de ocorrência do táxon.

Ocorrência nas amostras: BRASIL. Paraná: Pontal do Paraná, rio Guaraguaçu, 16/IV/2003, fitoplâncton, $P$. Bigunas, T. Ludwig \& A. Campos s.n. (UPCB47493); perifíton (UPCB47500); Pontal do Paraná, 24/X/2003, fitoplâncton, P. Bigunas, T. Ludwig \& J. Silva s.n. (UPCB47523).

\section{Eunotia sp. 3}

Fig. 70-73

Valvas com margem dorsal convexa; margem ventral reta a côncava, geralmente apresentando leve intumescimento próximo as extremidades valvares; extremidades atenuado-arredondadas, não destacadas do corpo valvar; estrias paralelas a radiadas; aréolas inconspícuas. Compr. 11,9-54,4 $\mu \mathrm{m}$; larg. 5,2-6,3 $\mu \mathrm{m}$; 14-15 estrias em $10 \mu \mathrm{m}$.

Alguns exemplares de maior comprimento de Eunotia sp. 3 assemelharam-se a duas espécies apresentadas por Berg (1939), principalmente com as figuras 69 e 72 c que correspondem a E. antiqua A. Berg e E. grunowi A. Berg, respectivamente. Estas espécies distinguem-se pela variação métrica superior às registradas nos exemplares do rio Guaraguaçu. E. antiqua apresenta entre 100-120 $\mu \mathrm{m}$ de comprimento, 7-8 $\mu \mathrm{m}$ de largura e 10-11,5 estrias em $10 \mu \mathrm{m}$, enquanto que E. grunowi tem entre 60-190 $\mu \mathrm{m}$ de comprimento e 10-12 estrias em $10 \mu \mathrm{m}$. Além disso, E. grunowi foi sinonimizada com E. valida Hustedt, e apesar dos exemplares encontrados estarem dentro da variação métrica apresentada por Hustedt (1927-1966), esta é ilustrada como tendo extremidades subcapitadas e ausência de intumescimento próximo às extremidades na margem ventral da valva.

Distribuição geográfica para o Estado do Paraná: primeira citação de ocorrência do táxon. 
Ocorrência nas amostras: BRASIL. Paraná: Pontal do Paraná, rio Guaraguaçu, 24/X/2003, fitoplâncton, P. Bigunas, T. Ludwig \& J. Silva s.n. (UPCB47516, 47519); perifíton (UPCB47515, 47518).
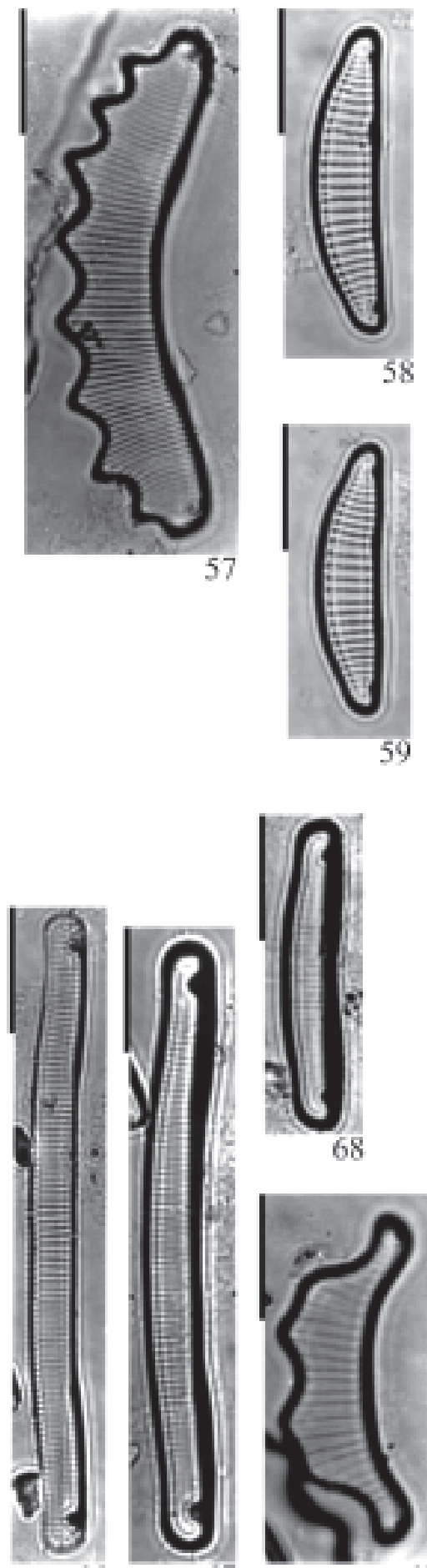

68

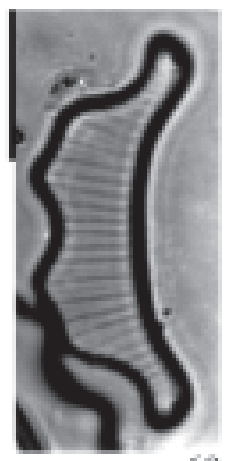

69
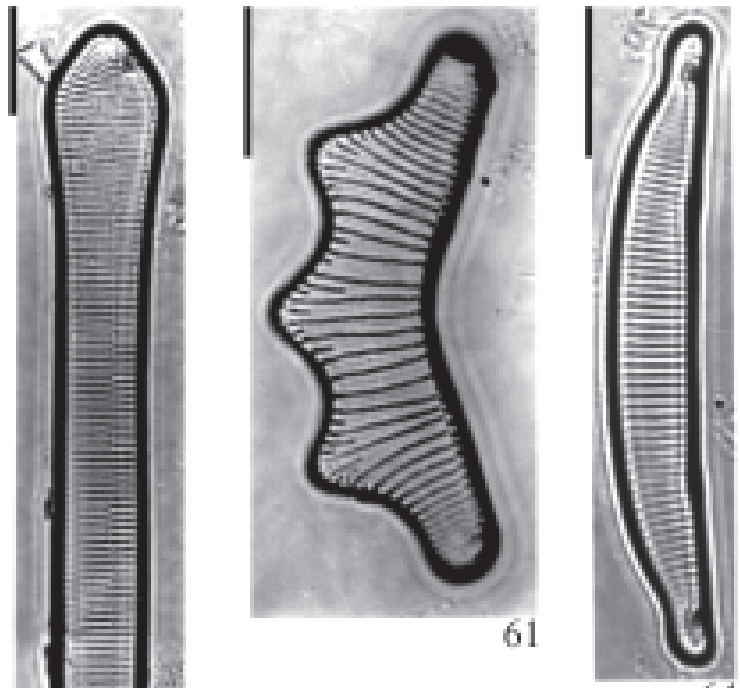

64
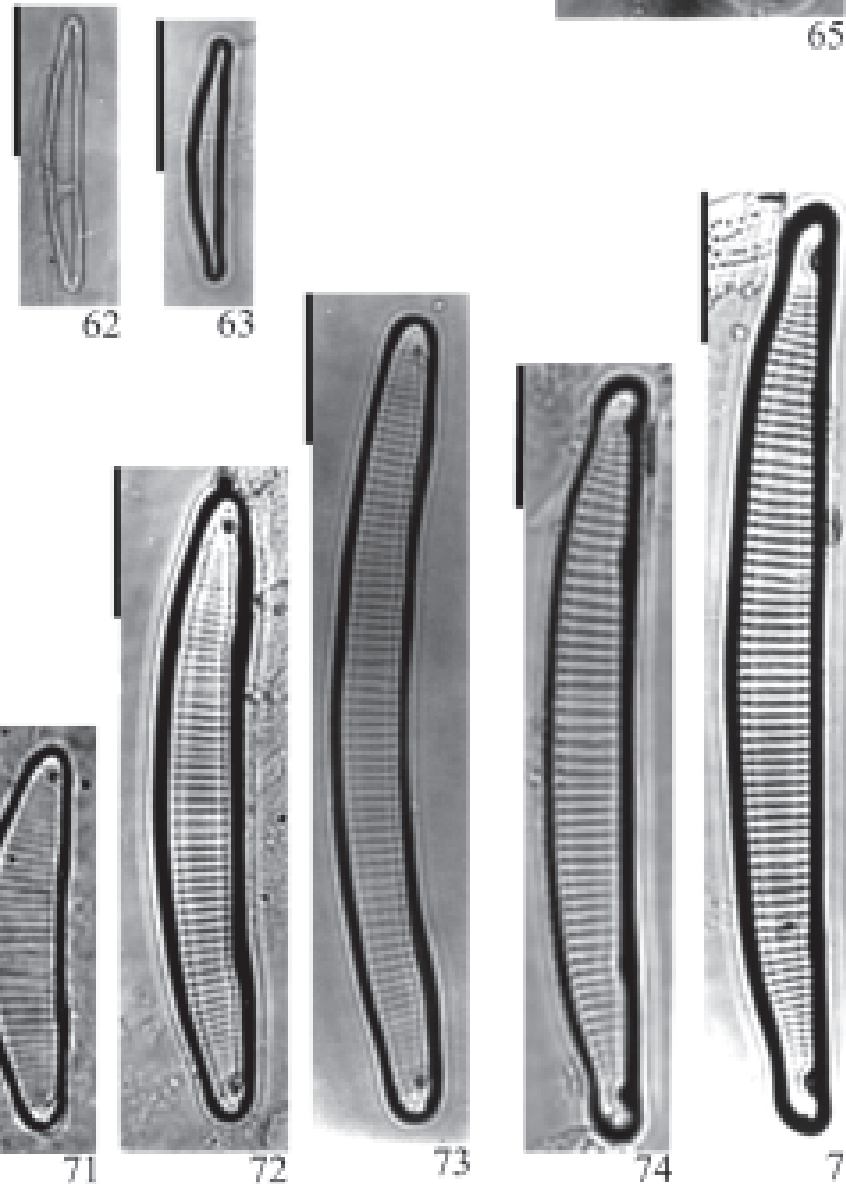

75

Figuras 57-75. Eunotia Ehrenberg (Bacillariophyceae) do rio Guaraguaçu, Paraná, Brasil. 57. Eunotia serra Ehrenberg. 58-59. Eunotia sudetica O. Müller. 60. Eunotia transfuga Metzeltin \& Lange-Bertalot. 61. Eunotia trigibba Hustedt. 62-63. Eunotia trinacria Krasske. 64. Eunotia veneris (Kützing) De Toni. 65. Eunotia zygodon Ehrenberg. 66-68. Eunotia sp. 1. 69. Eunotia sp. 2. 70-73. Eunotia sp. 3. 74-75. Eunotia sp. 4. Barras $=10 \mu \mathrm{m}$. 
subcapitado-atenuadas, destacadas do corpo valvar, mais estreitas que a largura mediana da valva; nódulos terminais nas extremidades; estrias paralelas a radiadas em direção às extremidades; aréolas inconspícuas. Compr.

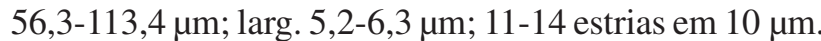

O táxon em questão apresentou semelhança com exemplares tanto de $E$. veneris quanto de $E$. pectinalis. Contudo, E. veneris apresenta margem dorsal mais fortemente convexa e maior densidade de estrias (18 estrias em $10 \mu \mathrm{m})$, e $E$. pectinalis margem dorsal reta a pouco convexa e extremidades mais largas e arredondadas que Eunotia sp. 4 (Carter \& Flower 1988; Hustedt 1927-1966). Exemplares similares a Eunotia sp. 4 foram registrados como Eunotia spec. N. 58/5-10 por Metzeltin \& Lange-Bertalot (1998) quando analisaram amostras brasileiras. Apesar de terem considerado o táxon distinto dos demais estudados, os autores não realizaram a proposição da nova espécie.

Distribuição geográfica para o Estado do Paraná: primeira citação de ocorrência do táxon.

Ocorrência nas amostras: BRASIL. Paraná: Pontal do Paraná, rio Guaraguaçu, 16/IV/2003, perifíton, P. Bigunas, T. Ludwig \& A. Campos s.n. (UPCB47500, 47502, 47503); fitoplâncton (UPCB47501); Matinhos, 24/X/2003, perifíton, P. Bigunas, T. Ludwig \& J. Silva s.n. (UPCB47510, 47511); Pontal do Paraná, 24/X/2003, fitoplâncton, P. Bigunas, T. Ludwig \& J. Silva s.n. (UPCB47512, 47514, 47516, 47519, 47523, 47524, 47525, 47526); perifíton (UPCB47513, 47515, 47517, 47518, 47520).

\section{Agradecimentos}

À Coordenação de Aperfeiçoamento de Pessoal de Nível Superior (CAPES), pela concessão de bolsa de Mestrado à primeira autora.

\section{Referências bibliográficas}

Barber, H.G. \& Harworth, E.Y. 1981. A Guide to the Morphology of the Diatom Frustule. Freshwater Biological Association. Ambleside, Cumbria.

Berg, A. 1939. Some new species and forms of the diatom genus Eunotia Her. 1837. Botaniska Notiser: 423-462.

Bicudo, D.C.; Morandi, L.L. \& Ludwig, T.A.V. 1999. Criptógamos do Parque Estadual das Fontes do Ipiranga, São Paulo, SP. Algas, 13: Bacillariophyceae (Eunotiales). Hoehnea 26: 173-184.

Bittencourt-Oliveira, M.C. 2002. A comunidade fitoplanctônica do rio Tibagi: uma abordagem preliminar de sua diversidade. Pp. 373402. In: M.E. Medri (ed.). A bacia do rio Tibagi, M.C. Gráfica.

Carter, J.R. \& Flower, R.J. 1988. A new species of Eunotia, E. pirla sp. nov., from Woolmer pond, an acid pool in the southeast of England. Diatom Research 3: 1-8.

Contin, L.F. 1990. Contribuição ao estudo das diatomáceas (Chrysophyta, Bacillariophyceae) na região da barragem de captação d'água do rio Iguaçu (SANEPAR), em Curitiba, Estado do Paraná, Brasil. Estudos de Biologia 24: 5-95.
De Oliveira, P.E. \& Steinitz-Kannan, M. 1992. The diatom flora (Bacillariophyceae) of the Cuyabeno Faunistic Reserve, Equatorian Amazonia. Nova Hedwigia 54: 515-552.

Ferrari, F.; Procopiak, L.K.; Alentar, Y.B. \& Ludwig, T.A.V. 2007. Eunotiaceae (Bacillariophyceae) em igarapés da Amazônia Central, Manaus e Presidente Figueiredo, Brasil. Acta Amazonica 37: $1-16$.

Frenguelli, J. 1941. Diatomeas del rio de La Plata. Revista del Museo de La Plata 3: 213-334.

Fürstenberger, C.B. \& Valente-Moreira, I.M. 2000. Diatomáceas (Bacillariophyta) perifíticas da lagoa Tarumã, Ponta Grossa, Paraná, Brasil. 2. Eunotiaceae. Insula 29: 117-134.

Hustedt, F. 1930. Bacillariophyta (Diatomeae). In: A. Pascher (ed.). Die Süsswasser-Flora Mitteleuropas. Jena: G. Fischer 10: $1-466$.

Hustedt, F. 1927-1966. Die Kieselalgen. In: Rabenhorst, L. Kryptogamen-Flora. Leipzig: Akademische Verlagsgesellschaft 7: 1-920, 1-845, 1-816.

Hustedt, F. 1965. Neue und wenig bekannte Diatomeen. IX. Süsswasserdiatomeen aus Brasilien, insbesondere des Amazonasgebietes. Internationale Revue der Gesamten Hydrobiologie und Hydrographie 59: 391-410.

Jensen, N.C. 1985. Hustedt's “Die Kieselalgen, 2. Teil": The Pennate Diatoms. Koenigstein, Koeltz Scientific Books.

Krammer, K. \& Lange-Bertalot, H. 1991. Bacillariophyceae: Centrales, Fragilariaceae, Eunotiaceae. In: H. Ettl; J Gerloff; H. Heynig \& D. Mollenhauer (eds.). Süsswasserflora von Mitteleuropa. Sttugart \& Jena, G. Fischer 2: 1-576.

Landucci, M. \& Ludwig, T.A.V. 2005. Diatomáceas de rios da bacia hidrográfica litorânea, PR, Brasil: Coscinodiscophyceae e Fragilariophyceae. Acta Botanica Brasilica 19: 345-357.

Lozovei, A.L. \& Luz, E. 1976. Diptera culicidae em Curitiba e arredores: II - alimentação. Arquivos de Biologia e Tecnologia 19: 43-83.

Lozovei, A.L. \& Shirata, M.T. 1990. Diatomáceas (Chrysophyta, Bacillariophyceae) no rio Passaúna, Curitiba, Paraná, Brasil Levantamento qualitativo da diatomoflórula em segmento de manancial. Estudos de Biologia 27: 5-56.

Ludwig, T.A.V. \& Flôres, T.L. 1995. Diatomoflórula dos rios da região a ser inundada para a construção da usina hidrelétrica de Segredo, PR. I. Coscinodiscophyceae, Bacillariophyceae (Achnanthales e Eunotiales) e Fragilariophyceae (Meridion e Asterionella). Arquivos de Biologia e Tecnologia 38: 631-650.

Ludwig, T.A.L. \& Valente-Moreira, I.M. 1989. Contribuição ao conhecimento da diatomoflórula do parque regional do Iguaçu, Curitiba, Paraná, Brasil: I. Eunotiaceae (Bacillariophyceae). Arquivos de Biologia e Tecnologia 32: 543-560.

Ludwig, T.A.V.; Bigunas, P.I.T.; Neiva, T.F.; Coquemala, V. \& Piccinini, C. 2005. Diatomáceas (Ochrophyta) dos lagos do Jardim Botânico. Revista do Museu Nacional do Rio de Janeiro 10: 301-323.

Metzeltin, D. \& Lange-Bertalot, H. 1998. Tropical diatoms of the South America I. Iconografia Diatomológica 5: 1-695.

Momoli, D.M.M. 1967. Contribuição ao estudo das diatomáceas do Tanque de Senegaglia, São José dos Pinhais, estado do Paraná, Brasil. Anais do Congresso da Sociedade de Botânica do Brasil 15: 33-46.

Moreira Filho, H. 1961. Flora de diatomáceas de planorbídeos de Curitiba. Pp. 130. In: Simpósio sobre bioquímica de planorbídeos.

Moreira Filho, H. \& Momoli, D.M.M. 1966. Diatomáceas em alguns focos larvários de anofelinos de Curitiba (Paraná, Brasil). Boletim da Universidade Federal do Paraná 15: 1-6.

Moreira Filho, H. \& Valente-Moreira, I.M. 1979. Diatomáceas da enseada da Prainha, Matinhos, Paraná. Boletim do Museu Botânico Municipal 35: 1-12.

Moreira Filho, H. \& Valente-Moreira, I.M. 1984. Catálogo das diatomáceas (Chrysophyta-Bacillariophyceae) marinhas e estuarinas do Estado do Paraná, Brasil. Acta Biologica Paranaense 13: 3-49. 
Moreira Filho, H. \& Valente-Moreira, I.M. 1981. Avaliação taxonômica e ecológica das diatomáceas (Bacillariophyceae) epífitas em algas pluricelulares obtidas nos litorais dos Estados do Paraná, Santa Catarina e São Paulo. Boletim do Museu Botânico Municipal 47: 1-17.

Moreira Filho, H., Valente-Moreira, I.M. \& Cecy, I.I.T. 1973. Diatomáceas na barragem de captação d'água (SANEPAR) do rio Iguaçu, em Curitiba, estado do Paraná. Acta Biologica Paranaense 2: 133-145.

Moreira Filho, H.; Cecy, I.I.T. \& Valente-Moreira, I.M. 1976. Diatomáceas da lagoa Dourada, estado do Paraná, Brasil. Tribuna Farmacêutica 44: 1-14.

Moro, R.S.; Garcia, E. \& Oliveira Júnior, H.F. 1994. Diatomáceas (Bacillariophyceae) da represa Alagados, Ponta Grossa, Paraná, Brasil. Iheringia 45: 5-19.

Patrick, R. 1940a. Diatoms of Northeastern Brazil. Part. I Coscinodiscophyceae, Fragilariophyceae and Eunotiaceae. Proceedings of the Academy of Natural Sciences of Philadelphia 92: 191-227.

Patrick, R. 1940b. Some new diatoms from Brazil. Notulae Nature 59: 1 -7.

Patrick, R. \& Reimer, C.W. 1966. The Diatoms of United States: exclusive of Alaska and Hawaii. Philadelphia: Academy of Natural Sciences 1: 1-688.

Reichardt, E. 1995. Die Diatomeen (Bacillariophyceae) in Ehrenberg's material von Cayenne, Guyana Gallica (1843). Iconographia Diatomologica 1: 1-99.

Round, F.E.; Crawford, R.M. \& Mann, D.G. 1990. The Diatoms: biology and morphology of the genera. New York, Cambridge University Press.

Sala, S.E.; Duque, S.R.; Núñez-Avellaneda, M. \& Lamaro, A.A. 2002. Diatoms from the Colombian Amazon: some species of the genus Eunotia (Bacillariophyceae). Acta Amazonica 32: 589-603.

Schmidt, A. 1874-1959. Atlas der Diatomaceen-Kunde. Reisland, Leipzing, O. R

Simonsen, R. 1974. The diatom plankton of the Indian Ocean Expedition of R/V "Meteor", 1964-65 "Meteor" Forschungsergbnisse, Reihe D-Biologie 19: 1-66.

Smayda, T.J. 1983. The phytoplankton of estuaries. In: B.H. Ketchum (ed.). Estuaries and enclosed seas. Ecosystems of the World 26: 65-102.
Souza, M.G.M. \& Compère, P. 1999. New diatoms species from the Federal District of Brazil. Diatom Research 14: 357-366.

Souza, M.G.M. \& Moreira-Filho, H.M. 1999. Diatoms (Bacillariophyceae) of two aquatic macrophyte banks from Lagoa Bonita, Distrito Federal, Brazil, I. Thalassiosiraceae and Eunotiaceae. Bulletin du Jardin Botanique National de Belgique 67: 259-278.

Souza-Mosimann, R.M.; Tavares, A.S. \& Freitas, V.P. 1997. Contribuição ao conhecimento da diatomoflórula do conteúdo estomacal de algumas espécies de peixes na Amazônia. I. Mileus sp. (Pacu) do lago do Prado, AM, Brasil. Acta Amazonica 27: $9-25$.

Tavares, B. \& Valente-Moreira, I.M. 2000. Diatomoflórula do lago de Cascavel, município de Cascavel, estado do Paraná, Brasil. Hoehnea 27: 1-24.

Torgan, L.C. \& Becker, V. 1997. Eunotia densistriata sp. nov.: a subaerial diatom from Southern Brazil. Diatom Research 12: $115-124$

Torgan, L.C. \& Becker, V. 1998. Eunotia itapuana, nom. nov. Diatom Research 13: 1 .

Torgan, L.C. \& Delani, O.M. 1988. Estudo taxonômico de diatomáceas (Bacillariophyceae) do "Complexo Banhado Grande", Rio Grande do Sul, Brasil: representantes do gênero Eunotia Ehrenberg. Iheringia, série Botânica 38: 81-107.

Valente-Moreira, I.M. 1975. Contribuição ao estudo das Bacillariophyceae (diatomáceas) em diatomitos brasileiros. Acta Biologica Paranaense 4: 135-198.

Valente-Moreira, I.T. \& Moreira Filho, H. 1982. Contribuição ao estudo das Bacillariophyceae (Diatomáceas) de Caiobá, Estado do Paraná. Acta Biologica Paranaense 11: 157-197.

Valente-Moreira, I.M.; Moreira Filho, H.M. \& Cunha, J.A. 1986. Diatomáceas (Chrysophyta, Bacillariophyceae) marinhas e estuarinas do Canal da Galheta, baía de Paranaguá, Paraná, Brasil. Acta Biologica Paranaense 19: 21-44.

Valente-Moreira, I.M.; Moreira Filho, H. \& Cunha, J.A. 1994. Diatomáceas (Chrysophyta, Bacillariophyceae) em biótopo do manguezal do rio Perequê, em Pontal do Sul, Paranaguá, Estado do Paraná, Estado do Paraná, Brasil. Acta Biologica Paranaense 23: 55-72. 